\title{
détermination en sondages de la perméabilité d'un milieu rocheux fracturé aspects théoriques et pratiques
}

\author{
par \\ L. Bertrand, E. Durand, B. Feuga \\ Bureau de Recherches Géologiques et Minières
}

\section{Introduction. Notion de milieu poreux équi- valent a un milieu fracturé}

Une grande partie des problèmes qui se posent en matière d'écoulement souterrain porte sur la détermination de la distribution des potentiels hydrauliques ou des débits dans les terrains.

Ces problèmes reçoivent des solutions en milieu poreux par recours à des méthodes de calcul faisant intervenir le tenseur de perméabilité des terrains, la définition de ce tenseur en tout point étant rendue possible par l'hypothèse de continuité du milieu. II est â noter que ces calculs permettent de déterminer des débits, ou des vitesses de filtration, qui s'en déduisent directement, et non pas des vitesses réelles des particules fluides.

Dans un milieu rocheux fracturé, l'essentiel de l'écoulement se fait par l'intermédiaire du réseau de fractures, et son caractère discontinu interdit normalement la définition d'un tenseur de perméabilité comme dans le cas d'un milieu poreux (continu).

La distinction entre milieu poreux. supposé continu, et milieu fracturé, supposé discontinu. est toutefois quelque peu arbitraire, et relève en fait d'une question d'échelle.

Considéré à grande échelle, en effet, le milieu poreux n'est pas continu puisque constitué de grains solides impermèables entre lesquels s'effectue l'écoulement. Ces grains peuvent être comparés aux blocs rocheux délimités par les fractures parcourant un massif, et, à petite échelle. ce massif fracturé peut lui-même être assimilé à un milieu poreux et continu.

On a coutume d'appeler ce milieu poreux fictif, de caractéristiques hydrauliques égales à celles du milieu fracturé qu'il représente, le milieu poreux équivalent (au milieu fracturé)

L'utilité de ce milieu poreux équivalent repose sur le fait que la relation flux-gradient de charge est linéaire aussi bien dans un milieu poreux que dans un milieu fracturé (en régime laminaire), ce qui rend possible son utilisation pour les calculs de distribution des charges hydrauliques et des débits moyens en milieu fracturé avec les méthodes mises au point pour les milieux poreux.

II va de soi que le recours au milieu poreux équivalent ne permet pas de résoudre tous les problèmes, et en particulier pas celui de la détermination des vitesses réelles d'écoulement dans les fractures; dans certains cas, la connaissance de ces vitesses est nécessaire et il faut alors recourir à des calculs prenant en compte les lois d'écoulement réelles dans les fissures, rien n'interdisant d'ailleurs de coupler les deux modes de calcul ou d'appuyer l'un sur l'autre.

L'assimilation, pour des raisons de facilité de calcul, d'un milieu fracturé à un milieu poreux étant, comme on l'a dit. une question d'échelle, il importe de savoir dans quelles conditions il peut y être recouru. On peut admettre que cette assimilation est justifiée si la maille de fracturation est petite par rapport à la dimension du volume du massif rocheux intéressé, y compris dans les zones de singularités éventuelles. C'est-à-dire que, par exemple, elle peut être faite dans le cadre d'études de ressources en eau: il peut néanmoins être nécessaire, dans un tel cas, d'individualiser, dans un éventuel modèle, les grands accidents structuraux tels que les failles, qu'iis constituent des barrières ou au contraire des drains.

L'écoulement de l'eau dans un milieu poreux est régi par l'équation de diffusivité

$$
\operatorname{div}(\vec{k} \cdot \overrightarrow{\operatorname{grad}} \phi)=s_{s} \cdot \frac{\partial \phi}{\partial t}
$$

\$ représentant la charge hydraulique $\mathrm{S}_{\mathrm{s}}$ l'emmagasinement spécifique du milieu et $\overrightarrow{\mathrm{k}}$ le tenseur de perméabilité.

Ce tenseur est caractérisé en tout point de l'espace par trois directions perpendiculaires (les directions principales de perméabilité dans le cas présent) auxquelles correspondent trois grandeurs numériques, les perméabilités principales. Les directions principales sont les trois seules directions de l'espace telles que la 
vitesse d'écoulement ait la même direction que le gradient de charge hydraulique. les facteurs de proportionnalité entre les deux n'étant autres que les perméabilités principales.

La dimension du tenseur de perméabilité ne peut être établie avec une certaine précision que par des essais à I'eau in situ aussi bien en milieu poreux qu'en milieu fracturé. De tels essais, réalisés dans plusieurs directions, peuvent d'ailleurs permettre également de déterminer la forme et l'orientation du tenseur

Ce dernier type d'essai est cependant couteux et de mise en œuvre difficile, et il ne peut y être recouru de manière systématique.

C'est pourquoi, partant du fait que les caractéristiques hydrodynamiques d'un milieu rocheux fracturé sont liées essentiellement à la fracturation (on supposera par la suite la matrice rocheuse imperméable), une méthode de détermination de la forme et de lorientation du tenseur des perméabilités à partir de données de fracturation (géologie structurale) a été mise au point.

La présente communication comporte deux parties. correspondant aux deux stades des études et travaux mis en œuvre pour la détermination des perméabilités d'un milieu rocheux fracturé

Dans la première est exposée la méthode utilisée pour la détermination de la forme (rapports d'anisotropie) et des directions principales du tenseur des perméabilités du milieu poreux équivalent, à partir de la connaissance des discontinuités du massif. Cette méthode repose sur une idée de Louis [1]. Dans la seconde sont présentées les mèthodes de mesure in situ de perméabilitès de massifs rocheux de très faible conductivité hydraulique qui ont été développées ces dernières années au B. R. G. M.

Dans la suite de cette communication, pour simplifier, on ne parlera plus de tenseur de perméabilité du milieu poreux équivalent au milieu rocheux fracturé, mais, de façon condensée, de tenseur de perméabilité du milieu rocheux fracturé.

De même, par facilité de langage, s'est-on autorisé à parler indifféremment de fractures ou de fissures. ces termes recouvrant d'ailleurs l'ensemble des types de discontinuités planaires pouvant affecter un massif rocheux et susceptibles d'être le siège de circulations d'eau.

2 Détermination de la forme et de l'orientation du tenseur des perméabilités d'un milieu rocheux fracturé à partir des données de l'analyse de la fracturation

\subsection{Approche théorique}

Considérons tout d'abord une fracture individuelle de conductivité hydraulique isotrope $\mathrm{k}$, définie par la relation

$\overrightarrow{\mathrm{v}}=-\mathrm{k} \cdot \overline{\mathrm{grad}} \phi \quad$ (régime laminaire)

$v=$ vitesse moyenne d'écoulement

$\phi=$ charge hydraulique (dans la formule ci-dessus, $\overrightarrow{\text { grad }} \phi$ représente en fait la projection du gradient de charge sur le plan de la fissure).

Soit un repère Oxyz lié à la fissure, tel que Oxy soit dans le plan de celle-ci, Ox étant horizontal.

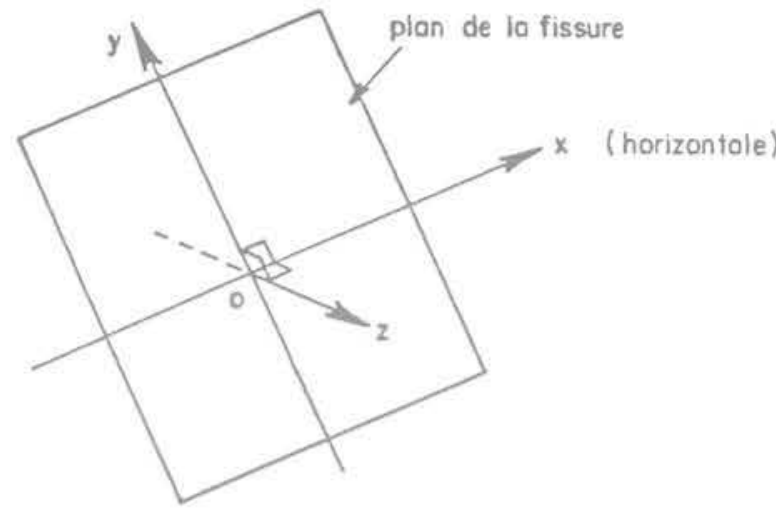

Dans ce repère. I'équation (2) s'écrit, sous forme matricielle

$$
\left\{\begin{array}{c}
v_{x} \\
v_{y} \\
v_{z}
\end{array}\right\}=\left\{\begin{array}{ccc}
k & 0 & 0 \\
0 & k & 0 \\
0 & 0 & 0
\end{array}\right\}\left\{\begin{array}{c}
\frac{a \phi}{i x} \\
\frac{i \phi}{\partial y} \\
\frac{a \phi}{i z}
\end{array}\right\}
$$

Soit maintenant un volume de référence du milieu rocheux fracturé, de forme cubique et de côté 1 .

Supposons que ce volume soit choisi de telle façon qu'il contienne la fissure considérée et que celle-ci soit parallèle à l'une de ses faces.

Considérons également le même volume du milieu poreux équivalent.

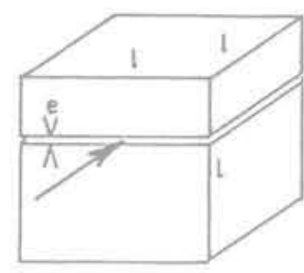

Milieu frocturé

(une fissure)

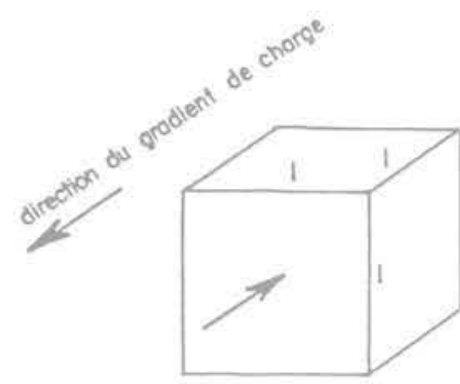

Milieu poreux équivolent
Le débit traversant la fissure est égal à

$$
\begin{aligned}
& \mathrm{Q}=\mathrm{v} \cdot 1 \cdot \mathrm{e}=1 \mathrm{ek}|\overline{\mathrm{grad}} \phi| \\
& (\mathrm{e}=\text { épaisseur de la fissure })
\end{aligned}
$$

Un mẽme débit doit traverser le volume du milieu poreux équivalent, pour lequel on a donc :

$$
\frac{Q}{S}=\frac{Q}{1^{2}}=k_{e q}|\overrightarrow{\operatorname{grad}} \phi|
$$

$k_{\text {eq }}$ étant la perméabilitè du milieu poreux équivalent dans la direction du plan de la fissure (cette perméabilité est nulle dans la direction perpendiculaire).

Le rapprochement de (4) et (5) donne

$$
\mathrm{k}_{\mathrm{eq}}=\frac{\mathrm{e}}{\mathrm{r}} \mathrm{k}
$$


La matrice représentant, dans le repère Oxyz, le tenseur de perméabilité du milieu poreux équivalent au volume du milieu fracturé contenant la fissure considérée s'écrit donc:

$$
K_{\text {eq }}=\frac{e}{1} k\left\{\begin{array}{lll}
1 & 0 & 0 \\
0 & 1 & 0 \\
0 & 0 & 0
\end{array}\right\}
$$

Considérons maintenant un repère $\mathrm{OXYZ}$ correspondant aux coordonnées géographiques : OX est orienté vers l'est, OY vers le nord et OZ suivant la verticale.

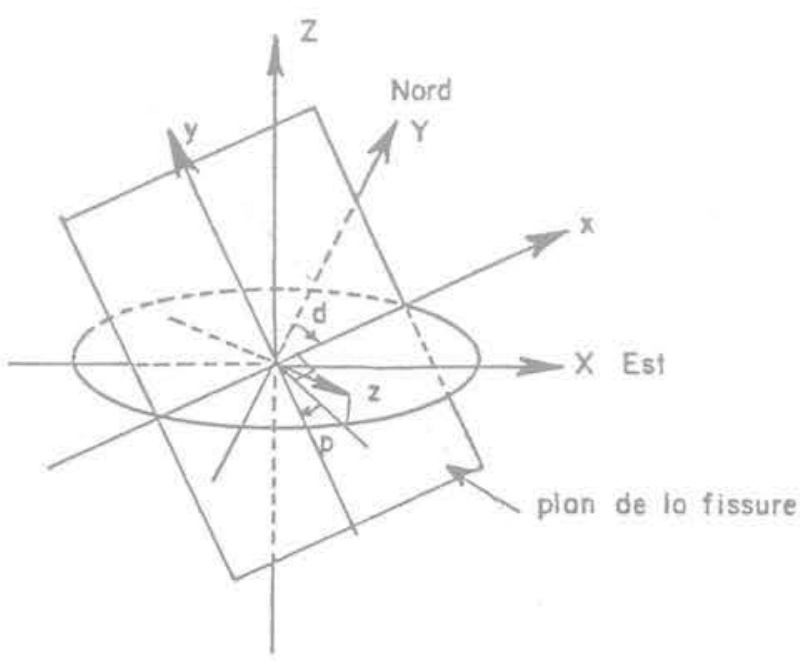

L'orientation du plan de fissure est définie par sa direction d par rapport au nord (convention de pendage à main droite) et par son pendage $p$.

La matrice de passage du repère Oxyz dans le repère géographique OXYZ s'écrit:

$$
Q=\left\{\begin{array}{lll}
\sin d & \cos d & 0 \\
-\cos d \cdot \cos p & \sin d \cdot \cos p & \sin p \\
\cos d \cdot \sin p & -\sin d \cdot \sin p & \cos p
\end{array}\right\}
$$

Dans le repére géographique OXYZ, la matrice représentant le tenseur de perméabilité du milieu poreux équivalent au volume du milieu fracturé contenant la fissure considérée s'écrit

$$
K_{\text {eq }}^{\prime}=Q^{1} \cdot K_{\text {eq }} \cdot Q
$$

ou encore, après développement des calculs

$$
\mathrm{K}_{\mathrm{eq}}=\frac{\mathrm{e}}{i} \mathrm{kR}
$$

avec

$$
R=\left\{\begin{array}{lll}
1-\cos ^{2} d \cdot \sin ^{2} p \frac{1}{2} \sin 2 d \cdot \sin ^{2} p & \frac{1}{2} \sin 2 p \\
\frac{1}{2} \sin 2 d \cdot \sin ^{2} p & 1-\sin ^{2} d \cdot \sin ^{2} p \frac{1}{2} \sin 2 p \\
-\frac{1}{2} \sin 2 p \cdot \cos d \frac{1}{2} \sin 2 p \cdot \sin d & \sin ^{2} p .
\end{array}\right\}
$$

Si on suppose maintenant que le volume de référence de milieu fracturé est recoupé non pas par une, mais par $\mathrm{N}$ fissures de conductivité hydraulique $k_{i}$, épaisseur $e_{i,}$ direction $d_{i}$ et pendage $p_{i,}$ i variant de 1 à $N$, le tenseur de perméabilité résultant du milieu poreux équivalent sera représenté, dans les coordonnées géographiques, par la somme des matrices correspon- dant aux différentes fissures individuelles. Cette sommation est justifiée par la linéarité de la loi liant le flux au gradient de charge*

$$
K_{\text {eq }}=\frac{N}{N} K_{\text {eq }}=\frac{1}{1} \sum_{1}^{N} e_{1} k_{1} R_{i}
$$

II est possible d'extraire les valeurs propres et les vecteurs propres de cette matrice, qui représenteront d'une part les perméabilités principales du milieu poreux équivalent et d'autre part les directions de ces perméabilités principales.

En pratique, si le relevé des orientations des discontinuités affectant un massif ne pose pas trop de problemes, pour peu que l'on dispose de zones oú ce massif se prête à l'observation (affleurements, galeries, sondages avec carottes orientées), il est par contre très difficile de déterminer l'épaisseur de ces discontinuités: les perturbations qui ont été imposées au massif rocheux pour amener à des conditions permettant son observation ont en effet presque toujours modifié les épaisseurs des fissures, et ce qui est observable dans ce domaine n'est en général pas représentatif de ce qui prévaut au sein du massif non perturbé: la décompression des terrains, les ébranlements dus aux tirs à l'explosif, le délavage par des circulations d'eau ou de fluides de forage ont pour effet d'ouvrir les fissures, et les perméabilités que l'on calcule en se fondant sur des épaisseurs observées dans de telles conditions sont toujours très supérieures aux perméabilités réelles déduites d'essais in situ intéressant des volumes de rocher non perturbés**

II est donc illusoire, en pratique, d'attendre de la formule (12) qu'elle permette de déterminer un module de perméabilité.

Par contre, elle permet. moyennant certaines hypothèses dont l'expérience acquise jusqu'à ce jour a montré qu'elles étaient justifiées, de calculer les valeurs relatives des perméabilitès principales (c'est-à-dire la forme du tenseur de perméabilité du milieu poreux équivalent) et les directions des perméabilités principales (c'est-à-dire l'orientation de ce tenseur)

L hypothèse la plus simple consiste à considérer que toutes les fissures sont identiques, c'est-à-dire que

et $\quad \begin{aligned} e_{1} & =e \\ k_{1} & =k\end{aligned}$

\section{quel que soit i.}

les valeurs de e et $k$ restant inconnues.

La recherche des vecteurs propres et des valeurs propres de la matrice $\frac{N}{i} R_{i}$ (le facteur constant $\frac{\text { ek }}{i}$ étant éliminé) donne des directions principales de perméabilité et des perméabilités principales qui n'ont de sens qu'en valeur relative.

\footnotetext{
- On suppose $\mathrm{N}$ assez grand pour que le calcul ait une valeur statistique et que linfluence de l hypothèse faite sur la forme cubique du volume de référence et le parallélisme de la fissure avec une de ses faces ne joue plus: 1 devient dès lors dans la formule (12) une simple longueur de référence dont la valeur $n$ 'a pas d'importance pratique, puisque l'effet de ses variations est très exactement compensé par celui des variations de $\mathrm{N}$ (dans l'hypothèse où la distribution spatiale de la fracturation, en densité et orientation. est la même en tout point. ce que nous admettons)

* La non continuité des fissures rèelles. non prise en compte en gènéral dans les calculs, explique également en partie l'écart entre perméabilités calculées et perméabilités mesurèes in situ
} 
Cette hypothèse peut néanmoins être contestée.

Il est alors possible de remplacer dans la formule (12) le facteur $e_{i} k_{i}$ par un poids $\delta_{i}$ déterminé au gré de l'utilisateur en fonction d'un certain nombre de paramètres caractérisant la fissure.

$\mathrm{Ce}$ poids est calculé, pour chaque fissure, à l'aide d'une formule de type

$$
\delta_{i}=f\left(t_{1}, t_{2}, t_{3}, t_{4}, \ldots\right)
$$

f étant une fonction, choisie par l'utilisateur. des paramètres $t_{1}, t_{2}, t_{3}, t_{4}$ etc. de la fissure. Ces paramètres peuvent être la continuité, l'ouverture libre, l'épaisseur apparente, l'état de contrainte mécanique dans le terrain (cet état de contrainte, qui joue sur l'ouverture de la fissure, est fonction en particulier de la profondeur), un indice relatif à d'éventuelles venues d'eau. la rugosité. le type de remplissage, etc.

De nombreux types de fonctions de pondération très différentes les unes des autres ont été testés sur plusieurs massifs. Les résultats obtenus, pour un même massif, n'ont jamais pour autant varié de façon significative, et ont en particulier toujours èté voisins de ceux obtenus en affectant le même poids à chaque fissure.

Ce résultat semble justifier cette méthode qui considere toutes les fissures comme indentiques du point de vue hydraulique. Cette hypothèse, qui est certes non fondée si lon considère chaque fissure prise individuellement, semble assez proche de la réalitè dès lors que l'on considère un grand nombre de fissures: la distribution des conductivités hydrauliques individuelles serait alors pratiquement la même pour toutes les familles de fractures considérées (une famille est constituée par l'ensemble des fissures qui se regroupent autour d'une orientation donnée, dès lors que le nombre de ces fissures représente un pourcentage significatif du nombre total de fissures relevées sur un site donnè).

II faut toutefois se garder d'ériger cette hypothèse, vérifiée jusqu'à présent sur tous les sites étudiés avec cette méthode, en régle générale, et il est dans tous les cas prudent de ne pas s'en contenter mais d'effectuer également des pondérations permettant de juger de sa validité. Des écarts importants entre les rèsultats obtenus avec et sans pondération doivent conduire à reprendre les données de terrain afin de déterminer la cause de cette différence et de juger lequel des résultats obtenus fournit la représentation la plus vraisemblable de la réalité.

\section{Remarque}

Parmi les pondérations que l'on peut ẻtre amené à effectuer, il en est une qui s'impose; c'est celle qui vise à corriger le biais introduit dans l'échantillon de fractures relevées sur une station par l'orientation propre de cette station. On concoit en effet que lors d'un levé on rencontre beaucoup plus de fractures appartenant à des familles d'orientation subperpendiculaire à celle de la station d'observation qu'appartenant à des familles qui lui sont subparallèles. La forme de la station, elle aussi, introduit un biais dans l'échantillon. II y a bien sûr lieu de corriger ces distorsions par des pondérations appropriées avant tout calcul du tenseur de perméabilité.

\subsection{Exemple d'application}

\subsubsection{Mine d'or d'Elsburg (Afrique du Sud)}

L'étude réalisée avait pour but de déterminer la direction optimale à donner à une galerie de liaison entre deux quartiers de la mine au niveau $-1500 \mathrm{~m}$ pour que cette galerie recoupe le moins de venues d'eau possible. Cette détermination nécessitait la connaissance de l'orientation du tenseur des perméabilités.

Les levès de fracturation réalisés dans les galeries accessibles au niveau -1500 ont permis de définir cinq grandes familles de discontinuités. pour chacune desquelles a été calculé un poids égal à la somme des poids individuels $\delta$ des éléments structuraux lui appartenant:

Les poids individuels $\delta$ des discontinuités ont été calculés à l'aide des formules suivantes:

Pour les failles: $\quad \delta=100$

Pour les diaclases : $\delta=10 \times c$

c représentant la continuité de l'élément structural (nombre variant de 0 à 1 . égal au rapport entre la Iongueur observable de cet élément et la longueur de lintersection du plan le contenant avec la paroi de la galerie)

Le tableau ci-dessous donne les directions, pendages et poids des différentes familles:

$\begin{array}{cccr}\text { Famille } n^{\circ} & \text { Direction } & \text { Pendage } & \text { Poids } \\ 1 & 106^{\circ} & 86^{\circ} & 8186 \\ 2 & 336^{\circ} & 87^{\circ} & 1323 \\ 3 & 204^{\circ} & 90^{\circ} & 696 \\ 4 & 147^{\circ} & 53^{\circ} & 447 \\ 5 & 181^{\circ} & 68^{\circ} & 182\end{array}$

A partir de ces valeurs, il a été possible de calculer la matrice représentant le tenseur de perméabilité du milieu poreux équivalent à chaque famille de discontinuités, en considérant chacune d'elles comme une seule fissure et en utilisant la formule 10, le facteur $\frac{\mathrm{ek}}{\mathrm{t}}$ de la formule étant pris égal au poids de la famille. On a ainsi obtenu les matrices suivantes:

Famille 1 $\quad\left\{\begin{array}{rrr}7566 & -2160 & 157 \\ -2160 & 823 & -541 \\ 157 & -541 & 8153 \\ 221 & -491 & -63 \\ -491 & 1104 & 28 \\ -63 & 28 & 1320 \\ 115 & 258 & 0 \\ 258 & 580 & 0 \\ 0 & 0 & 696\end{array}\right\}$
Famille 2
Famille 3 $\quad\left\{\begin{array}{rrr}247 & -130 & 179 \\ -130 & 362 & -116 \\ 179 & -116 & 285 \\ 26 & 3 & 63 \\ 3 & 182 & 1 \\ 63 & 1 & 157\end{array}\right\}$
dont la somme n'est autre que la matrice représentant le tenseur de perméabilité du milieu poreux équivalent à lensemble du milieu rocheux fracturé dans les coordonnées géographiques :

\begin{tabular}{|c|c|c|c|}
\hline & $E-W$ & $N-S$ & Verticale, \\
\hline$E-W$ & 8175 & -2520 & $336)$ \\
\hline N-S & -2520 & 3051 & -628 \\
\hline Verticale & 336 & -628 & 10611 \\
\hline
\end{tabular}

Rappelons que les coefficients de cette matrice n'ont de sens qu'en valeur relative. 
L'extraction de ses valeurs et vecteurs propres a donné les résultats suivants:

\begin{tabular}{|c|c|c|c|c|}
\hline & \multicolumn{2}{|c|}{ Valeur propre } & \multicolumn{2}{c|}{ Vecteur propre } \\
\hline & valeur absolue & valeur relative & Direction & Pendage \\
\hline 1 & 10821 & 5,42 & $301^{\circ}$ & $71^{\circ}$ \\
2 & 9021 & 4.52 & $111^{\circ}$ & $19^{\circ}$ \\
3 & 1995 & 1 & $202^{\circ}$ & $3^{\circ}$ \\
\hline
\end{tabular}

Ces résultats sont représentés de façon schématique sur les figures 1 et 2 .

On voit qu'il existe une anisotropie de perméabilitè très marquée, de l'ordre de 5 , entre la direction $200^{\circ}$ (horizontale) et le plan vertical de direction $110^{\circ}$ qui constitue un plan de perméabilité préférentielle.

II est clair que la galerie devait être parallèle à cette direction de plan pour rencontrer le moins de venues d'eau possible.

\subsubsection{Mine de diamant de Kimberley (Afrique du Sud)}

Les résultats prèsentés ci-après ont été acquis dans le cadre d'une étude du drainage de la mine à ciel ouvert de Kimberley ("Big Holew)

Les levés structuraux ont été réalisés dans une galerie concentrique á la fosse. sous $60 \mathrm{~m}$ de recouvrement. sur neuf portions de galerie de $30 \mathrm{~m}$ de long réparties tout autour de la fosse. Les données ont été traitées

\section{DIAGRAMME DE SCHMIDT}

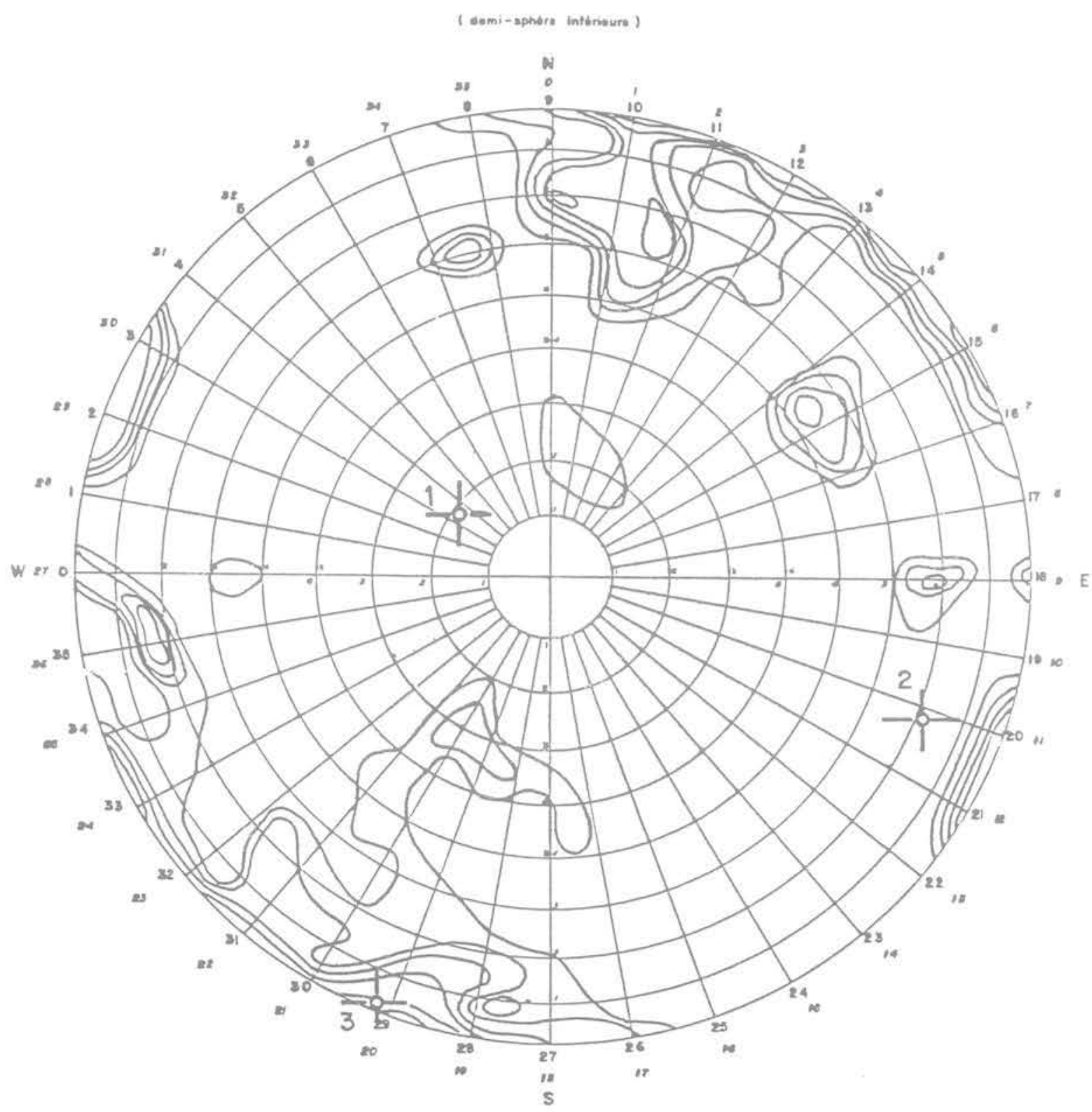

Fig. 1 Mine d'or d'Elsburg - Représentation de la fracturation (avec pondération) et des directions principales de perméabilité 


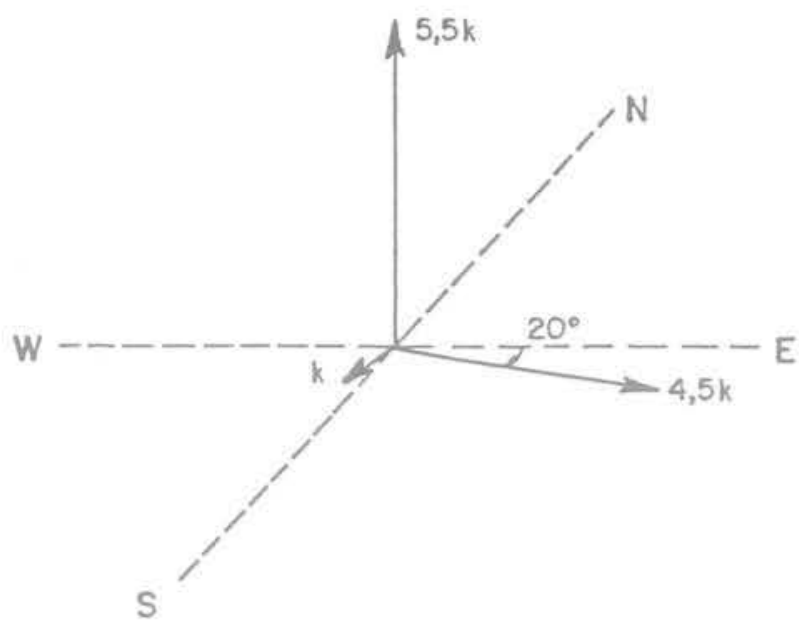

Fig. 2 Mine d'or d'Elsburg - Représentation simplifiée du tenseur de perméabilité du milieu directement, pour chacune des neuf stations delevés, à l'aide d'un programme d'ordinateur. CPFA, qui fournit les directions et les valeurs des perméabilités principales.

La figure 3 présente les résultats obtenus pour l'une des stations. sur un diagramme de Schmidt oủ sont figurés par ailleurs les nuages de points correspondant aux pôles des éléments structuraux relevés.

Le tableau I récapitule l'ensemble des résultats obtenus pour les neuf stations. Ces résultats sont par ailleurs représentés de façon schématique sur la figure 4 , en surimposition sur un plan de la mine.

Une première constatation que l'on peut faire est que l'anisotropie est nettement plus faible que dans le site d'Elsburg traité ci-dessus (maximum 4,28; valeur moyenne aux alentours de 2), Une telle anisotropie n'a que très peu d'influence sur l'écoulement dans le massif qui est pratiquement le même que si ce dernier était isotrope.

\section{DIAGRAMME DE SCHMIDT}

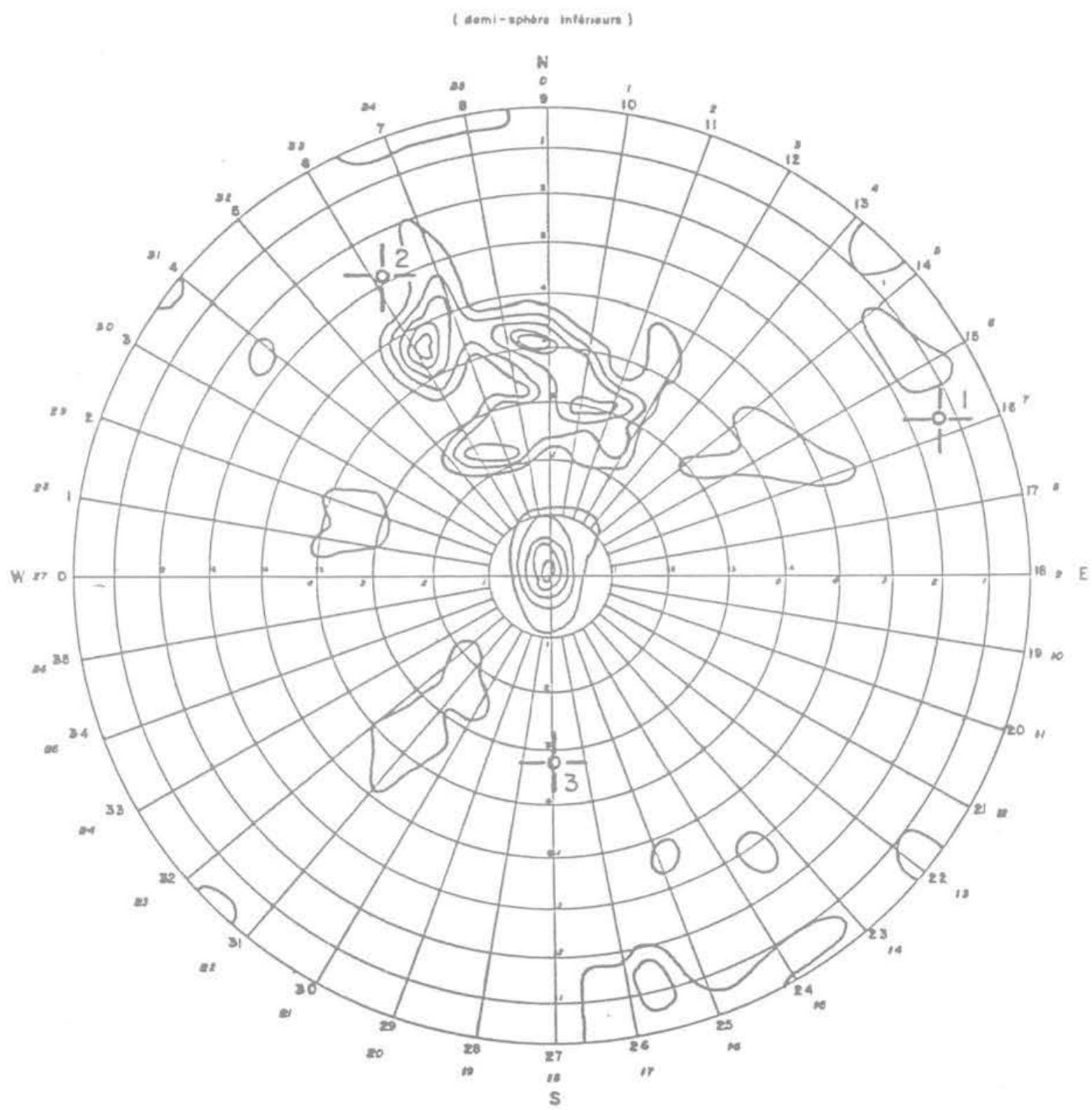

Fig. 3 Mine de diamant de Kimberley - Représentation de la fracturation pour la station 7 (sans pondération) et des directions principales de perméabilité 
Tableau 1

Mine de Kimberley

Directions principales de perméabilité

aux différentes stations de levés structuraux

\begin{tabular}{|c|c|c|c|}
\hline \multirow{2}{*}{$\begin{array}{c}\text { Numéro de } \\
\text { station }\end{array}$} & \multicolumn{2}{|c|}{ Directions principales de perméabilité } & \multirow{2}{*}{$\begin{array}{l}\text { Valeurs relatives } \\
\text { des perméabilités } \\
\text { (anisotropie) }\end{array}$} \\
\hline & Direction & Pendage & \\
\hline \multirow[t]{3}{*}{1} & $139^{\circ}$ & $82^{\circ}$ & 2,99 \\
\hline & $287^{\circ}$ & $7^{\circ}$ & 2,26 \\
\hline & $18^{\circ}$ & $4^{\circ}$ & 1 \\
\hline \multirow[t]{3}{*}{2} & $242^{\circ}$ & $46^{\circ}$ & 2,49 \\
\hline & $74^{\circ}$ & $44^{\circ}$ & 2,12 \\
\hline & $338^{\circ}$ & $6^{\circ}$ & 1 \\
\hline \multirow[t]{3}{*}{3} & $246^{\circ}$ & $77^{\circ}$ & 1,92 \\
\hline & $34^{\circ}$ & $11^{\circ}$ & 1,30 \\
\hline & $125^{\circ}$ & $7^{\circ}$ & 1 \\
\hline \multirow[t]{3}{*}{4} & $114^{\circ}$ & $82^{\circ}$ & 1,71 \\
\hline & $272^{\circ}$ & $7^{\circ}$ & 1.22 \\
\hline & $2^{\circ}$ & $3^{\circ}$ & 1 \\
\hline \multirow[t]{3}{*}{5} & $329^{\circ}$ & $55^{\circ}$ & 1,76 \\
\hline & $143^{\circ}$ & $35^{\circ}$ & 1,51 \\
\hline & $235^{\circ}$ & $3^{\circ}$ & 1 \\
\hline \multirow{3}{*}{6} & $115^{\circ}$ & $23^{\circ}$ & 1.64 \\
\hline & $286^{\circ}$ & $67^{\circ}$ & 1,41 \\
\hline & $23^{\circ}$ & $3^{\circ}$ & 1 \\
\hline \multirow[t]{3}{*}{7} & $67^{\circ}$ & $13^{\circ}$ & 3,99 \\
\hline & $330^{\circ}$ & $29^{\circ}$ & 3.39 \\
\hline & $179^{\circ}$ & $58^{\circ}$ & 1 \\
\hline \multirow[t]{3}{*}{8} & $266^{\circ}$ & $2^{\circ}$ & 1.99 \\
\hline & $359^{\circ}$ & $52^{\circ}$ & 1.50 \\
\hline & $175^{\circ}$ & $38^{\circ}$ & 1 \\
\hline \multirow[t]{3}{*}{9} & $299^{\circ}$ & $32^{\circ}$ & 4,28 \\
\hline & $89^{\circ}$ & $54^{\circ}$ & 3.25 \\
\hline & $200^{\circ}$ & $14^{\circ}$ & 1 \\
\hline
\end{tabular}

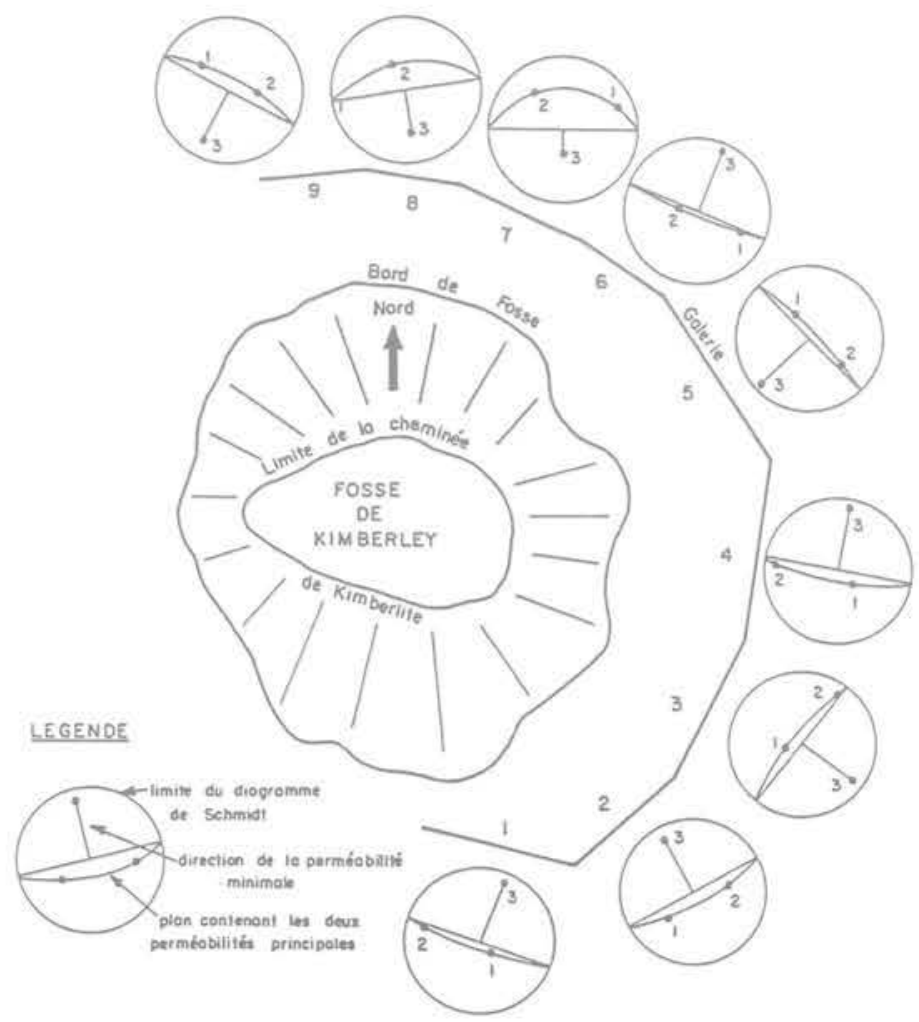

Fig. 4 Représentation schématique de la fosse de Kimberley (à $1 / 5000$ ) et du tenseur de perméabilité autour de cette fosse 


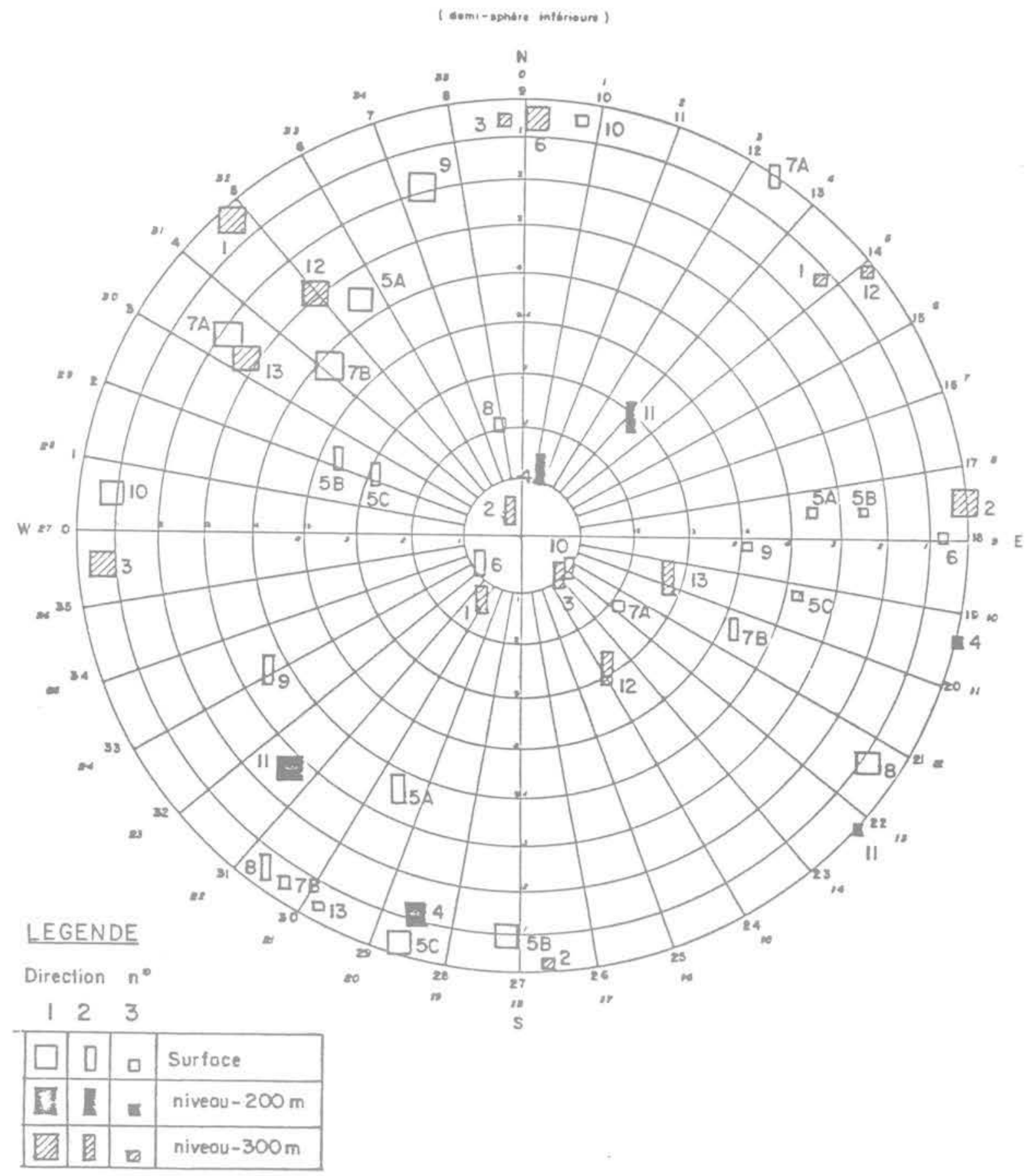

Fig. 5 Mine de cuivre de Murdochville - Représentation des directions principales de perméabilité pour les différentes stations de relevés

L'autre constatation importante est que pour la plupart des stations, le plan de perméabilité maximale est un plan vertical à peu près perpendiculaire au rayon vecteur mené depuis le centre de la fosse. L'orientation des perméabilitès est donc en gros axisymétrique, ce qui semble pouvoir être mis sur le compte de la structure géologique qui est elle même axisymétrique (la mine exploitait une cheminée verticale de kimberlite recoupant des terrains horizontaux).

II s'agit là d'un exemple intéressant d'influence directe de la géologie sur la physionomie des perméabilités d'un massif.

\subsubsection{Mine de cuivre de Murdochville (Québec)}

Les résultats présentés ci-après ont été obtenus, comme les précédents, dans le cadre d'une étude de drainage d'une mine à ciel ouvert. Le gisement, de type stockwerk, est développé autour d'une intrusion porphyrique recoupant des séries sédimentaires à dominante argileuse et gréseuse, transformées localement en quartzites intensément fracturées.

Les levés structuraux ont èté effectués en surface, sur les gradins de la mine, et dans des galeries de reconnaissance du gisement à environ $200 \mathrm{~m}$ et $300 \mathrm{~m}$ sous le niveau du sol naturel.

Le tableau ci-après présente les résultats obtenus (sans pondération). 


\begin{tabular}{|c|c|c|c|c|c|c|c|c|c|c|}
\hline \multirow{2}{*}{$\begin{array}{l}\mathrm{N}^{\circ} \text { de } \\
\text { station }\end{array}$} & \multirow{2}{*}{ Localisation } & \multicolumn{3}{|c|}{ Direction 1} & \multicolumn{3}{|c|}{ Direction 2} & \multicolumn{3}{|c|}{ Direction 3} \\
\hline & & Dir. & Pend. & $\begin{array}{c}\text { Perm. } \\
\end{array}$ & Dir. & Pend: & Perm. & Dir. & Pend. & Perm. \\
\hline $5 \mathrm{~A}$ & Surface & $326^{\circ}$ & $35^{\circ}$ & 29,3 & $204^{\circ}$ & $36^{\circ}$ & 19,5 & $175^{\circ}$ & $35^{\circ}$ & 1 \\
\hline $5 B$ & Surface & $181^{\circ}$ & $10^{\circ}$ & 1.88 & $291^{\circ}$ & $61^{\circ}$ & 1,65 & $175^{\circ}$ & $26^{\circ}$ & 1 \\
\hline $5 \mathrm{C}$ & Surface & $196^{\circ}$ & $3^{\circ}$ & 1.53 & $291^{\circ}$ & $53^{\circ}$ & 1.33 & $102^{\circ}$ & $37^{\circ}$ & 1 \\
\hline $7 \mathrm{~A}$ & Surface & $304^{\circ}$ & $21^{\circ}$ & 1,64 & $34^{\circ}$ & $2^{\circ}$ & 1.23 & $126^{\circ}$ & $68^{\circ}$ & 1 \\
\hline $7 B$ & Surface & $310^{\circ}$ & $42^{\circ}$ & 3.23 & $116^{\circ}$ & $47^{\circ}$ & 1,72 & $214^{\circ}$ & $6^{\circ}$ & 1 \\
\hline 8 & Surface & $123^{\circ}$ & $9^{\circ}$ & 1.95 & $216^{\circ}$ & $6^{\circ}$ & 1,77 & $337^{\circ}$ & $79^{\circ}$ & 1 \\
\hline 9 & Surface & $343^{\circ}$ & $18^{\circ}$ & 2,10 & $241^{\circ}$ & $36^{\circ}$ & 1,61 & $92^{\circ}$ & $48^{\circ}$ & 1 \\
\hline 10 & Surface & $275^{\circ}$ & $9^{\circ}$ & 1,70 & $129^{\circ}$ & $79^{\circ}$ & 1,63 & $7^{\circ}$ & $5^{\circ}$ & 1 \\
\hline 4 & Niveau -200 & $195^{\circ}$ & $11^{\circ}$ & 2.02 & $15^{\circ}$ & $78^{\circ}$ & 1,83 & $104^{\circ}$ & $0^{\circ}$ & 1 \\
\hline 11 & Niveau -200 & $223^{\circ}$ & $27^{\circ}$ & 3,09 & $42^{\circ}$ & $62^{\circ}$ & 2,35 & $132^{\circ}$ & $1^{\circ}$ & 1 \\
\hline 1 & Niveau -300 & $317^{\circ}$ & $4^{\circ}$ & 1,59 & $212^{\circ}$ & $75^{\circ}$ & 1,06 & $48^{\circ}$ & $14^{\circ}$ & 1 \\
\hline 2 & Niveau -300 & $85^{\circ}$ & $2^{a}$ & 8,62 & $329^{\circ}$ & $85^{\circ}$ & 8,43 & $176^{\circ}$ & $3^{\circ}$ & 1 \\
\hline 3 & Niveau -300 & $266^{\circ}$ & $7^{\circ}$ & 1.98 & $129^{\circ}$ & $81^{\circ}$ & 1.79 & $356^{\circ}$ & $6^{\circ}$ & 1 \\
\hline 6 & Niveau -300 & $1^{\circ}$ & $6^{\circ}$ & 2,90 & $232^{\circ}$ & $80^{\circ}$ & 2.36 & $90^{\circ}$ & $8^{\circ}$ & 1 \\
\hline 12 & Niveau -300 & $319^{\circ}$ & $29^{\circ}$ & 2,49 & $149^{\circ}$ & $61^{\circ}$ & 1,64 & $141^{\circ}$ & $3^{\circ}$ & 1 \\
\hline 13 & Niveau -300 & $302^{\circ}$ & $27^{\circ}$ & 2.35 & $106^{\circ}$ & $63^{\circ}$ & 1,79 & $208^{\circ}$ & $5^{\circ}$ & 1 \\
\hline
\end{tabular}

Ces résultats sont également représentés sur la figure 5 .

Deux conclusions principales ont pu être tirées de I'étude réalisée:

- l'anisotropie de permèabilité des terrains est presque partout très faible:

- le seul trait caractéristique de la distribution des directions principales de perméabilité est la distinction entre la surface et les terrains en profondeur: en surface, bien que la densité de fracturation varie considérablement d'une station à l'autre, la distribution des directions de perméabilité semble quelconque, et. globalement, on peut considérer que le tenseur est isotropé. En profondeur, au contraire, le plan dans lequel les perméabilités sont les plus èlevées est subvertical, d'orientation moyenne SE-NW. Ce plan est iuimẽme pratiquement isotrope, la perméabilité dans la direction perpendiculaire étant environ deux fois plus faible.

2.2.4 Les trois exemples qui ont été présentés portent sur des terrains situés à des profondeurs différentes : $1500 \mathrm{~m}$ à Elsburg, $60 \mathrm{~m}$ à Kimberley, 0 à $300 \mathrm{~m}$ à Murdochville.

Bien que les contextes géologiques de chacun de ces trois exemples soient trè différents les uns des autres. il est néanmoins intéressant d'examiner comment varient l'anisotropie de perméabilité d'une part et le pendage du plan des perméabilités maximales d'autre part, en fonction de la profondeur.

Ces informations sont rassemblées dans le tableau ci-dessous :
On constate, á la vue de ce tableau, que plus la profondeur augmente, plus le plan des perméabilités maximales tend à devenir vertical et plus l'anisotropie augmente.

Des considérations mécaniques permettent sans doute d'expliquer partiellement cette tendance : alors qu'en surface le faible état des contraintes dans les terrains permet lapparition de cassures dans toutes les directions (en particulier des fractures de décompression parallèles à la surface du sol), en profondeur, au contraire, la contrainte verticale due au poids des terrains, qui est la plupart du temps nettement supérieure aux contraintes horizontales, empêche ou limite fortement lapparition de cassures horizontales: seules apparaissent alors des fractures subverticales ou obliques, qui donnent lieu à des perméabilités verticales supérieures aux perméabilités horizontales.

Ceci ne suffit cependant pas à expliquer une anisotropie de Fordre de 5 entre les perméabilités contenues dans un plan vertical et la perméabilité dans la direction horizontale perpendiculaire: une telle anisotropie ne peut s'expliquer que par une densité beaucoup plus forte de fractures subparallèles au plan de perméabilités maximales, cette densité s'expliquant peut-être elle-mẻme par une anisotropie du champ des contraintes horizontales (il s'agit évidemment d'une explication simpliste, la plupart des massifs rocheux ayant été soumis à plusieurs épisodes cassants successifs correspondant à des champs de contrainte différents: l'explication donnée serait alors valable pour la phase cassante ayant donné lieu à la fracturation la plus intense)

\begin{tabular}{|l|c|c|c|}
\hline \multicolumn{1}{|c|}{ Mine } & Profondeur & $\begin{array}{c}\text { Pendage moyen des } \\
\text { plans des perméabilités } \\
\text { maximales }\end{array}$ & $\begin{array}{c}\text { Anisotropie } \\
\text { moyenne }\end{array}$ \\
\hline Elsburg & $1500 \mathrm{~m}$ & $87^{\circ}$ & 4.97 \\
Murdochville & $300 \mathrm{~m}$ & $83^{\circ}$ & $1,99^{*}$ \\
Murdochville & $200 \mathrm{~m}$ & $89^{\circ}$ & 2.32 \\
Kimberley & $60 \mathrm{~m}$ & $75^{\circ}$ & 2,26 \\
Murdochville & $0 \mathrm{~m}$ & $52^{\circ}$ & $1,78^{*}$ \\
\hline
\end{tabular}

\footnotetext{
- Ces chiffres ne tiennent pas compte des valeurs extrérnes. considérées comme non représentatives.
} 


\section{Mesures de perméabilité en sondages en milieu rocheux très peu perméable}

\subsection{Principe des mesures}

La plus répandue des méthodes de mesure in situ des perméabilités des terrains est sans conteste le pompage d'essai en puits ou sondage.

Cette méthode permet de déterminer une transmissivité, définie théoriquement comme le produit de la perméabilité des terrains, supposés homogènes, par l'épaisseur de la couche aquifère. Bien évidemment. même en milieu alluvionnaire, il est rare que la perméabilité des terrains soit constante suivant la verticale, et le pompage d'essai, s'il permet d'évaluer une perméabilité moyenne, ne permet pas de déterminer le profil des perméabilités.

Or la connaissance de ce profil est nécessaire pour la résolution de bon nombre de problèmes, particulièrement en milieu rocheux fracturé, où lhypothèse de constance de la perméabilité suivant la verticale n'a aucune chance d'être vérifièe. même dans une formation homogène du point de vue lithologique, et ce pour au moins deux raisons

La première est que la perméabilité des milieux fracturés dépend essentiellement de l'ouverture des fractures qui les recoupent ${ }^{*}$, et que cette ouverture est fonction de l'état de contrainte mécanique dans le terrain. qui augmente proportionnellement à la profondeur.

La seconde est liée à l'hétérogénéité de la distribution des fractures, non pas tant en orientation qu'en densité. En particulier, il est rare qu un massif rocheux ne soit pas recoupé par quelques "grands " accidents á proximité desquels la densité de fracturation est beaucoup plus intense que dans les zones plus éloignées

Un autre inconvénient des pompages d'essai est qu'ils se prêtent mal à la mesure de très faibles perméabilités. en raison des risques de dénoyage des pompes, du fait de rabattements trop forts. même à très faible débit, et des problèmes matériels et théoriques (effet de capacité prépondérant) que pose l'utilisation de trés faibles débits en pompage. Une méthode qui ne présente ni l'un ni l'autre de ces inconvénients consiste à réaliser, au lieu de pompages, des injections d'essai entre obturateurs. Cette méthode permet d'isoler, dans un sondage, des zones limitées sur lesquelles un examen minutieux des carottes permet de conclure que la perméabilité ne varie pas ou varie peu

D'autre part, comme n'existe pas, en ce qui concerne la pression d'injection, la limitation qui existe pour le rabattement, il est possible d'imposer au niveau de la passe de sondage testee un gradient de charge hydraulique très supérieur à celui que peut créer un pompage.

Dans son principe, la méthode n'est pas nouvelle, puisqu'elle a été développée après la dernière guerre, en particulier en France par Maurice Lugeon, qui a donné son nom à un essai normalisé d'injectabilité des terrains. Toutefois, ce type d'essai n'a pas pour but la mesure des perméabilités des terrains, mais l'évalua-

- La conductivité hydraulique d'une fracture individuelle est proportionnelle au carré de l'ouverture de celle-ci. tion de leur capacité à absorber des coulis d'injection. dans le cas de fondations de barrages en particulier. Ils intéressent une épaisseur limitée de terrains assez perméables.

La méthode a été perfectionnée, sur les plans théoriques et pratiques, par un certain nombre d'ingénieurs, afin de permettre la détermination des perméabilités des massifs rocheux. Néanmoins, la plupart des applications récentes restaient limitées à une profondeur d'investigation assez faible et à des pressions peu élevées. Ce n'est que récemment que le B.R.G.M. a développé une technologie permettant de travailler à grande profondeur, dans des sondages de petit diamètre*, et sous des pressions élevées, portant ainsi le seuil des perméabilités mesurables à l'aide d'injections d'essaì entre obturateurs de $10^{-8}$ à $10^{10} \mathrm{~m} / \mathrm{s}$.

3.2 Possibilités de la méthode de mesure des perméabilités en sondage par injections entre obturateurs

Avant d'aborder les aspects théoriques et matériels de la méthode, il apparait intéressant de donner un aperçu de ses possibilités et de ses modalités d'utilisation actuelles.

C'est l'objet du tableau II, qui résume les principales caractéristiques d'un certain nombre de campagnes de mesures récentes, réalisées dans les conditions les plus variées.

La méthode a été utilisée avec succès dans des terrains très différents, allant de terrains très résistants à des terrains de faible tenue (schistes houillers), la réalisation d'essais dans ces derniers présentant évidemment des difficultés beaucoup plus grandes et nécessitant des précautions particulières.

La profondeur d'essai maximale est à ce jour de $540 \mathrm{~m}$. mais dans de bons terrains la méthode peut sans difficulté être mise en œuvre à des profondeurs doubles ou triples. Comme on peut le voir également, la méthode a déjá été utilisée dans des sondages de très petit diamètre $(49 \mathrm{~mm})$, et dans des trous inclinés.

Enfin, la gamme de perméabilités mesurées est très large (de $10^{-4}$ à $2 \cdot 10^{-10} \mathrm{~m} / \mathrm{s}$ ), le matériel d'injection et de mesure devant bien évidemment être adapté en fonction de la valeur des perméabilités à mesurer.

\subsection{Types d'essais. Méthodes d'interprétation}

Les essais peuvent être conduits de deux manières différentes : soit à pression constante, soit à débit constant.

\subsubsection{Essais à pression constante}

Dans ce cas, on impose dans la chambre dinjection une surpression maintenue constante, et on enregistre les variations du débit injecté, jusqu'à stabilisation. On passe ensuite à une pression supérieure, elle aussi maintenue constante jusqu'à stabilisation du débit et ainsi de suite. On répète ensuite la même séquence d'opérations, mais cette fois par paliers de pression décroissants.

- L'industrie pétrolière possède des techniques lui permettant éalement de travailler à grande profondeur. mais dans des sondages de gros diamétre. 
Tableau II

Principales caractéristiques de quelques campagnes de mesure de perméabilité en sondage

\begin{tabular}{|c|c|c|c|c|c|c|c|c|}
\hline Localisation & $\begin{array}{l}\text { Mine de } \\
\text { cuivre } \\
\text { Québec }\end{array}$ & $\begin{array}{l}\text { Kimberley } \\
\text { mine } \\
\text { Atr. du Sud }\end{array}$ & $\begin{array}{l}\text { Koffiefontein } \\
\text { mine } \\
\text { Afr. du Sud }\end{array}$ & $\begin{array}{l}\text { Mayet de } \\
\text { montagne } \\
\text { (Allier) }\end{array}$ & $\begin{array}{l}\text { Mine de } \\
\text { Faulquemont } \\
\text { (Moselle) }\end{array}$ & $\begin{array}{c}\text { Granite } n^{\circ} 1 \\
\text { France }\end{array}$ & $\begin{array}{c}\text { Granite } n^{\circ} 2 \\
\text { France }\end{array}$ & Portugal \\
\hline Géologie & $\begin{array}{c}\text { "Porphyre } \\
\text { cuprifere } \\
\text { quartzite }\end{array}$ & $\begin{array}{l}\text { Dolérite } \\
\text { et schistes }\end{array}$ & Granite & Granite & $\begin{array}{l}\text { Schistes } \\
\text { et charbon }\end{array}$ & Granite & Granite & $\begin{array}{c}\text { Schistes } \\
\text { et } \\
\text { grauwackes }\end{array}$ \\
\hline $\begin{array}{c}\text { Nombre de } \\
\text { torages }\end{array}$ & 6 & 6 & 3 & 1 & $\begin{array}{c}1 \\
\text { (incliné } \\
\text { à } 30^{\circ} \text { ) }\end{array}$ & 1 & 1 & 7 \\
\hline $\begin{array}{c}\text { Diamétre des } \\
\text { forages }\end{array}$ & $60 \mathrm{~mm}$ & $165 \mathrm{~mm}$ & $49 \mathrm{~mm}$ & $126 \mathrm{~mm}$ & $96 \mathrm{~mm}$ & $101 \mathrm{~mm}$ & $96 \mathrm{~mm}$ & 72 a $86 \mathrm{~mm}$ \\
\hline $\begin{array}{l}\text { Profondeur } \\
\text { maximale }\end{array}$ & $425 \mathrm{~m}$ & $64 \mathrm{~m}$ & $250 \mathrm{~m}$ & $155 \mathrm{~m}$ & $316 \mathrm{~m}$ & $101 \mathrm{~m}$ & $540 \mathrm{~m}$ & $360 \mathrm{~m}$ \\
\hline $\begin{array}{l}\text { Longueur } \\
\text { passe } \\
\text { injection }\end{array}$ & $6 \mathrm{~m}$ & $\begin{array}{l}4 m \\
2 m\end{array}$ & $5 \mathrm{~m}$ & $3 m$ & $4.50 \mathrm{~m}$ & $1 \mathrm{~m}$ & $10 \mathrm{~m}$ & $10 \mathrm{~m}$ \\
\hline $\begin{array}{c}\text { Type } \\
\text { d'obturateur }\end{array}$ & Mazier & Mazier & $\begin{array}{c}\text { Mazier et } \\
\text { Pétrométalic }\end{array}$ & Lynes & TAM & $\begin{array}{c}\text { Manchettes } \\
\text { sonde triple } \\
\text { Louis }\end{array}$ & TAM & TAM \\
\hline $\begin{array}{c}\text { Matériel } \\
\text { d'injection }\end{array}$ & $\begin{array}{l}\text { Pompe à } \\
\text { piston }\end{array}$ & $\begin{array}{c}\text { Pompe } \\
\text { centrifuge }\end{array}$ & $\begin{array}{l}\text { Pompe à } \\
\text { piston } \\
\text { (injection) }\end{array}$ & $\begin{array}{l}\text { Pompe } \\
\text { à piston }\end{array}$ & Surpresseur & $\begin{array}{l}\text { Pompe } \\
\text { centrifuge }\end{array}$ & $\begin{array}{c}\text { Pompe } \\
\text { Triplex et } \\
\text { surpresseur }\end{array}$ & $\begin{array}{c}\text { Pompe à } \\
\text { piston } \\
\text { et } \\
\text { surpresseur }\end{array}$ \\
\hline $\begin{array}{c}\text { Matériel de } \\
\text { mesure }\end{array}$ & $\begin{array}{l}3 \text { débitm. } \\
2 \text { mano. }\end{array}$ & $\begin{array}{l}\text { Compt. vol. } \\
2 \text { mano. }\end{array}$ & Manos. & $\begin{array}{c}\text { Capteur P. } \\
\text { compteur } \\
\text { volum. }\end{array}$ & $\begin{array}{l}2 \text { mano. } \\
\text { compteur } \\
\text { volum. }\end{array}$ & $\begin{array}{l}2 \text { compteurs } \\
\text { volum. } \\
\text { Mano. }\end{array}$ & $\begin{array}{l}3 \text { héliflu } \\
1 \text { capteur } \mathrm{P} \\
\text { compt. vol. }\end{array}$ & $\begin{array}{l}2 \text { compteurs } \\
\text { volum. } \\
2 \text { mano }\end{array}$ \\
\hline $\begin{array}{l}\text { Type } \\
\text { d'essai }\end{array}$ & $\begin{array}{l}\text { Paliers de } \\
\text { pression }\end{array}$ & $\begin{array}{c}\text { Paliers de } \\
\text { pression }\end{array}$ & $\begin{array}{c}\text { Paliers de } \\
\text { débit }\end{array}$ & $\begin{array}{c}\text { Paliers de } \\
\text { débit }\end{array}$ & $\begin{array}{l}\text { Paliers de } \\
\text { pression }\end{array}$ & $\begin{array}{l}\text { Paliers de } \\
\text { pression }\end{array}$ & $\begin{array}{l}\text { Paliers de } \\
\text { pression }\end{array}$ & $\begin{array}{l}\text { Paliers de } \\
\text { pression }\end{array}$ \\
\hline $\begin{array}{l}\text { Pression } \\
\text { mini } \\
\text { maxi }\end{array}$ & $\begin{array}{c}\text { (MPa) } \\
0.1 \\
1.5\end{array}$ & $\begin{array}{c}(\mathrm{MPa}) \\
0.07 \\
1.0\end{array}$ & $\begin{array}{c}\text { (MPa) } \\
1 \\
18\end{array}$ & $\begin{array}{c}(\mathrm{MPa}) \\
0.001 \\
1,5\end{array}$ & $\begin{array}{c}\text { (MPa) } \\
0,3 \\
8,0\end{array}$ & $\begin{array}{c}(\mathrm{MPa}) \\
0,05 \\
1.2\end{array}$ & $\begin{array}{c}(\mathrm{MPa}) \\
0.2 \\
10.5\end{array}$ & $\begin{array}{c}(\mathrm{MPa}) \\
0,3 \\
4,0\end{array}$ \\
\hline $\begin{array}{l}\text { Débit } \\
\text { mini } \\
\text { maxi }\end{array}$ & $\begin{array}{c}(\ell / m n) \\
0.01 \\
100\end{array}$ & $\begin{array}{c}\text { (1/mn) } \\
0.5 \\
90\end{array}$ & $\begin{array}{c}(\ell / m n) \\
10 \\
60\end{array}$ & $\begin{array}{c}(\ell / \mathrm{mn}) \\
12,0 \\
33,0\end{array}$ & $\begin{array}{c}(\ell / \mathrm{mn}) \\
0,01 \\
10,0\end{array}$ & $\begin{array}{c}(f / m n) \\
0,1 \\
80\end{array}$ & $\begin{array}{c}(t / m n) \\
0.02 \\
5.0\end{array}$ & $\begin{array}{c}(\ell / \mathrm{mn}) \\
0,4 \\
98,0\end{array}$ \\
\hline $\begin{array}{c}\text { Perméabilité } \\
\text { mini } \\
\text { maxi }\end{array}$ & $\begin{array}{c}(\mathrm{m} / \mathrm{s}) \\
5 \cdot 10^{-10} \\
10^{-5}\end{array}$ & $\begin{array}{c}(\mathrm{m} / \mathrm{s}) \\
2 \cdot 10^{-7} \\
2 \cdot 10^{-5}\end{array}$ & $\begin{array}{c}(\mathrm{m} / \mathrm{s}) \\
2 \cdot 10^{-\mathrm{s}} \\
10^{-6}\end{array}$ & $\begin{array}{c}(\mathrm{m} / \mathrm{s}) \\
0,1 \cdot 10^{-6} \\
0,4 \cdot 10^{-6}\end{array}$ & $\begin{array}{c}(\mathrm{m} / \mathrm{s}) \\
3 \cdot 10^{-10} \\
2 \cdot 10^{-7}\end{array}$ & $\begin{array}{c}(\mathrm{m} / \mathrm{s}) \\
10^{-7} \\
1.1 \cdot 10^{-4}\end{array}$ & $\begin{array}{c}(\mathrm{m} / \mathrm{s}) \\
2 \cdot 10^{-10} \\
4 \cdot 10^{-9}\end{array}$ & $\begin{array}{c}(\mathrm{m} / \mathrm{s}) \\
3 \cdot 10^{-10} \\
1.6 \cdot 10^{-7}\end{array}$ \\
\hline
\end{tabular}

L'une des deux formules suivantes, valables pour un régime d'écoulement permanent, permet de calculer la perméabilité pour tout couple surpression $\Delta p$, débit stabilisé $Q$ :

Écoulement horizontal (dans le plan perpendiculaire au sondage)

$\mathrm{K}=\frac{\mathrm{Q}}{2 \pi \mathrm{L} \cdot \Delta \mathrm{p}} \log \left(\frac{\mathrm{Ri}}{\mathrm{rp}}\right)$

Écoulement tridimensionnel

$K=\frac{Q}{2 \pi L \cdot \Delta p} \log \left(\frac{L}{r p}\right)$

$\mathrm{L}=$ longueur de la passe d'injection

$r p=$ rayon du sondage

$\mathrm{Ri}=$ rayon d'influence, notion empirique correspondant à la distance à laquelle la charge hydraulique dans le terrain n'est pas modifiée par l'essai. Ce rayon d'influence, en général inconnu et que l'on doit donc estimer, et par ailleurs très critiquable sur le plan théorique n'a en fait pas une très grande incidence puisqu'il intervient dans un logarithme.
Dans le cas idéal, la courbe Q. $\Delta p$ est une droite de pente proportionnelle à K (fig. 6).

Dans la réalité, très souvent cette courbe n'est pas une droite, et, en outre, il est trés fréquent qu'elle ne soit pas la même à la montée et à la descente en pression. ce qui traduit un comportement apparemment non réversible des fractures. En réalité, cette nonréversibilité apparente provient dans bien des cas du fait que la stabilisation du débit ne correspond pas à l'établissement d'un véritable régime permanent, et que de ce fait la distribution des charges hydrauliques dans le massif n'est pas la mëme, pour une pression d'injection donnée, suivant que la chambre d'injection n'a pas encore été soumise à une pression supérieure ou suivant qu'elle l'a été une courte période de temps auparavant. Cette non-réversibilité est en principe d'autant plus marquée que les fissures sont moins perméables.

Quant à la non-linéarité de la courbe $Q\lrcorner$.$p , elle est de$ règle dès que l'on dépasse une certaine pression d'injection entraînant une ouverture élastique des 


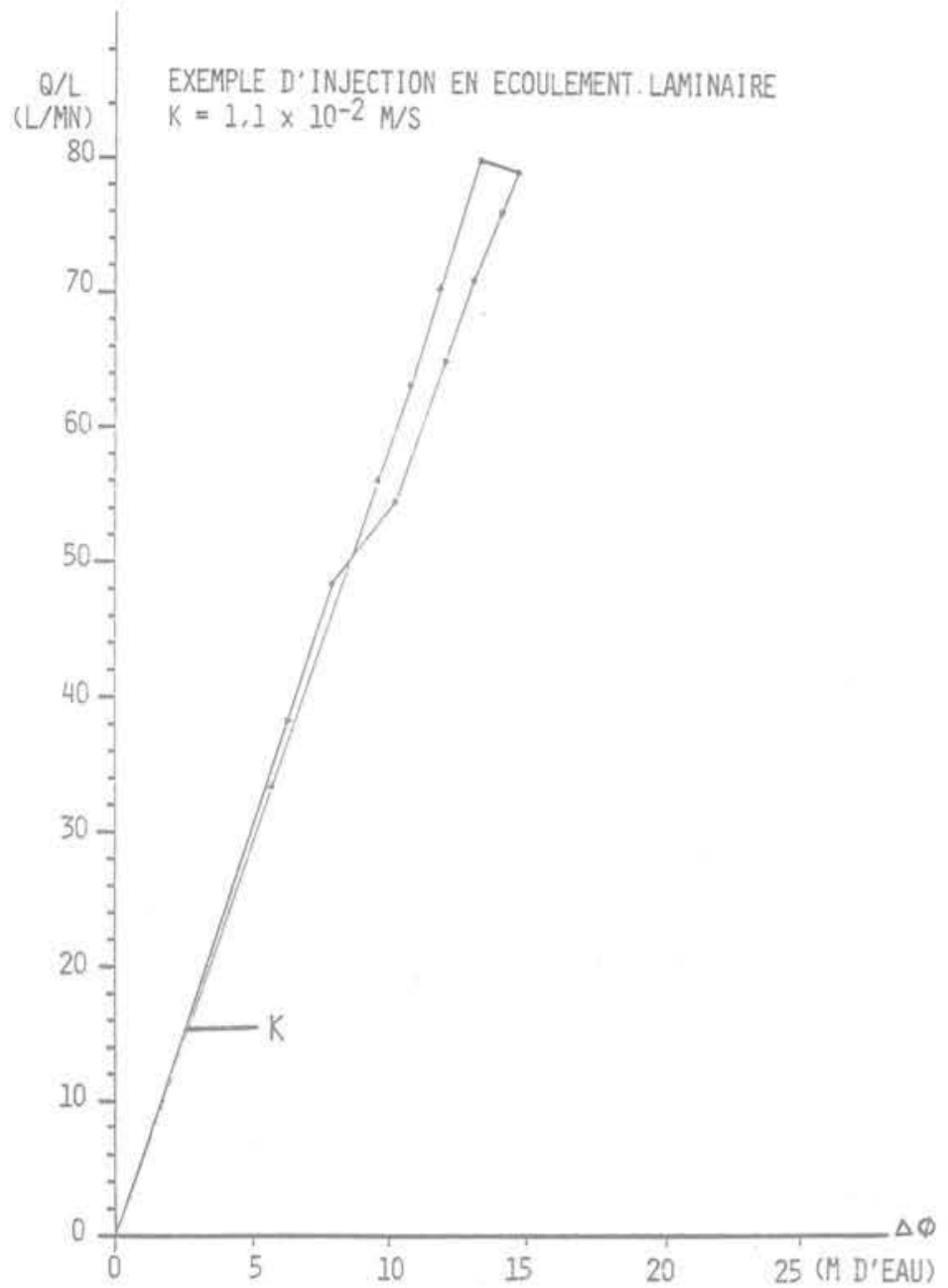

Fig. 6 Comportement réversible et linéaire d'un milieu fissuré (profondeur : $20 \mathrm{~m}$ )

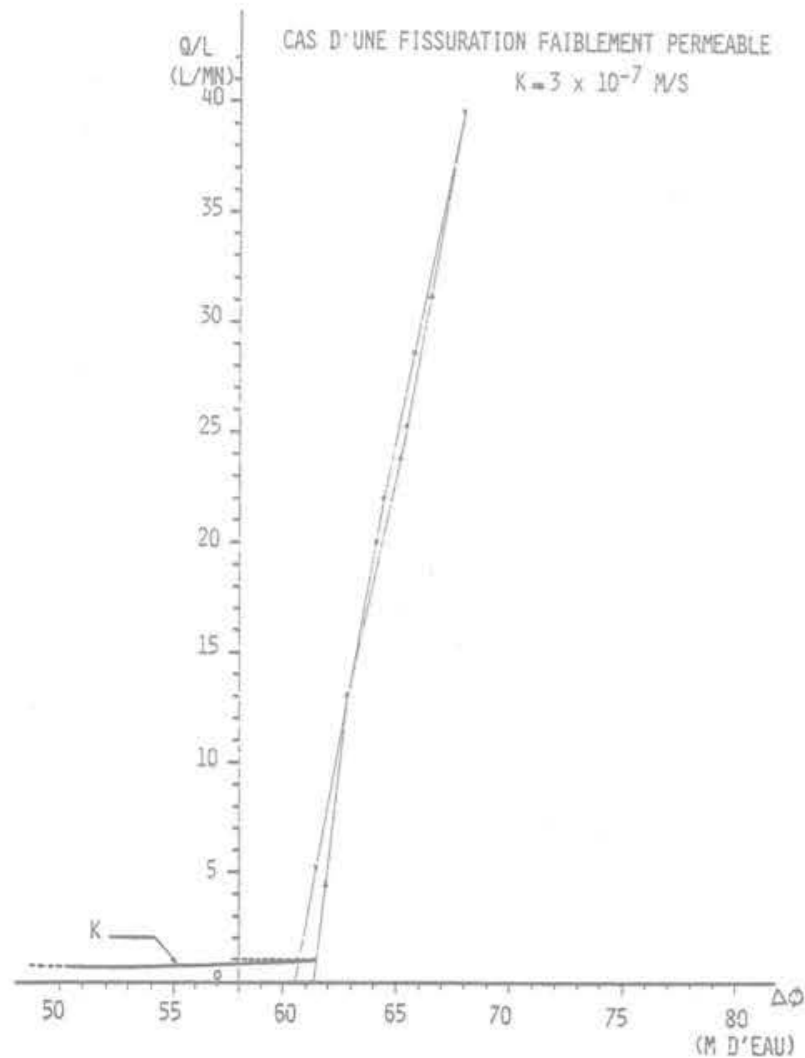

Fig. 7 Comportement réversible mais non linéaire d'un. milieu fissuré (profondeur : $60 \mathrm{~m}$ ) fissures, et donc une augmentation de leur conductivité hydraulique. II faut noter toutefois que l'ouverture de certaines fissures peut entraîner la fermeture d'autres et que des schémas trop simplistes ne permettent pas toujours d'interpréter les courbes obtenues.

Une pression trop élevée, excédant en un point la contrainte principale mineure, peut en outre claquer le terrain, ouvrant des fissures nouvelles ou étendant des fractures existantes d'extension limitèe. Dans ce cas. la non-réversibilité du comportement des fissures entre la montée et la descente en pression est évidemment réelle.

Un exemple caractéristique de non-linéarité est offert par la figure 7 oú une fissure fermée et peu perméable se trouve brusquement ouverte à partir d'une certaine pression et voit sa conductivité augmenter brutalement dans des proportions trés importantes, son comportement restant toutefois réversible, ce qui témoigne d'une ouverture élastique et non d'un claquage.

\subsubsection{Essais à débit constant}

Le principe des injections d'essai à dèbit constant est le même que celui des pompages d'essai.

Un débit constant est injecté dans la chambre d'injection pendant une certaine durée, l'évolution de la pression d'injection étant enregistrée pendant l'essai. Après arrêt de l'injection, la chambre d'injection est isolée et on enregistre la chute de pression avec le temps.

Ce type d'essai, lors duquel le régime d'écoulement est transitoire, peut être interprété à l'aide des modèles théoriques propres aux milieux poreux (Theis. Jacob. Boulton, etc. [2] [3] [4])

Des modèles plus spécifiques, prenant en compte I'existence de fractures ont été développés par Gringarten et Ramey [5], puis par Thiery [6]. Ces modèles, très utilisès dans la pratique, ont toutefois un domaine d'utilisation limité aux faibles pressions d'injection, n'entrainant pas une modification de la géométrie du milieu (ouverture des fissures) et donc de la perméabilité.

Les figures 8 et 9 représentent les variations de pression en injection et en décharge (chute de pression après injection) pour des essais réalisés à une centaine de mètres de profondeur dans une passe de sondage recoupée par une fracture verticale. Ce genre de courbe est très classique pour des essais de cette nature. En figure 10, on a représenté la courbe-type, établie par Gringarten et Ramey pour ce genre de configuration.

On peut constater que les courbes expérimentales sont très éloignées de la courbe-type et que cette dernière est de peu de secours pour interpréter les résultats des essais.

3.3.3. En fait, et ceci est vrai également pour les essais à pression constante, seuls des modèles prenant en compte le couplage des phénomènes hydrauliques et des phénomènes mécaniques dans le milieu fissuré permettraient de rendre compte des phénomènes observés lors des essais d'injection au-delà d'un certain seuil de pression, au demeurant assez peu élevé (et fonction évidemment de la profondeur).

De tels modèles font l'objet de recherches dans de nombreux pays, et en particulier en France, mais il est peu probable, compte tenu du grand nombre de 


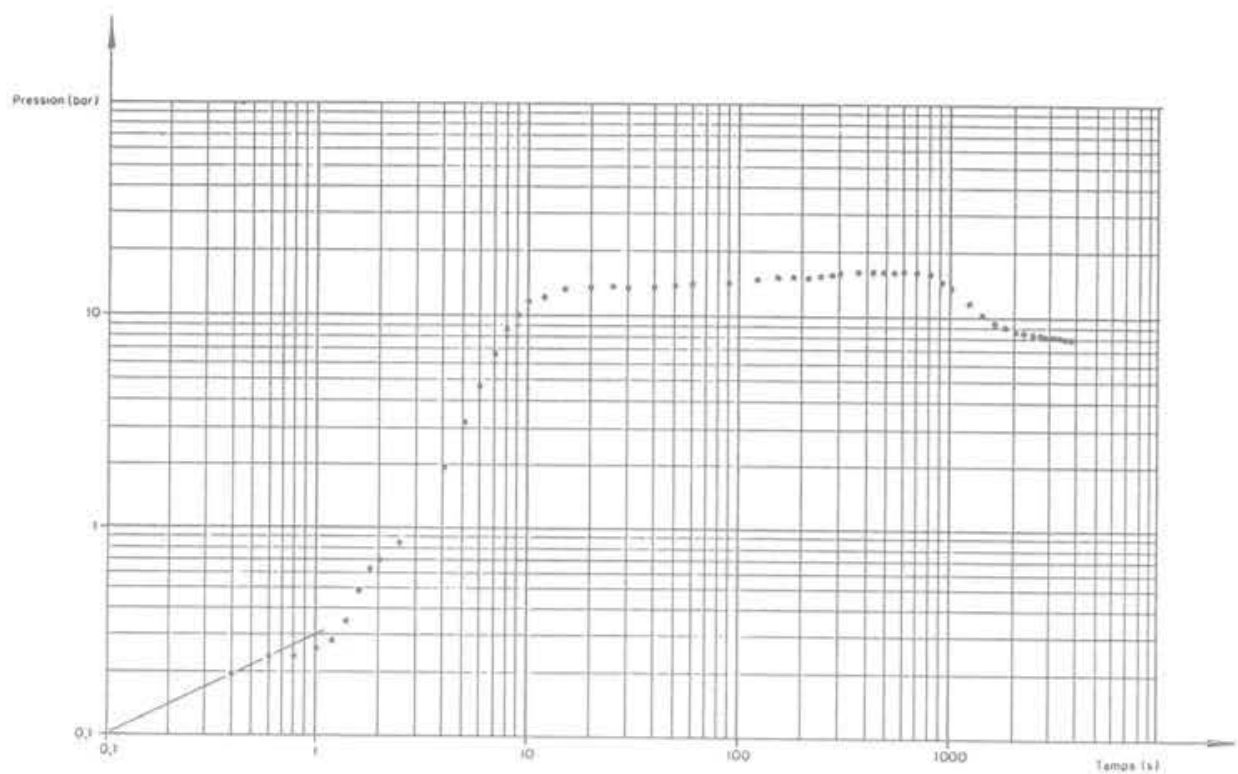

Fig. 8 Exemple d'injection dans une fracture verticale

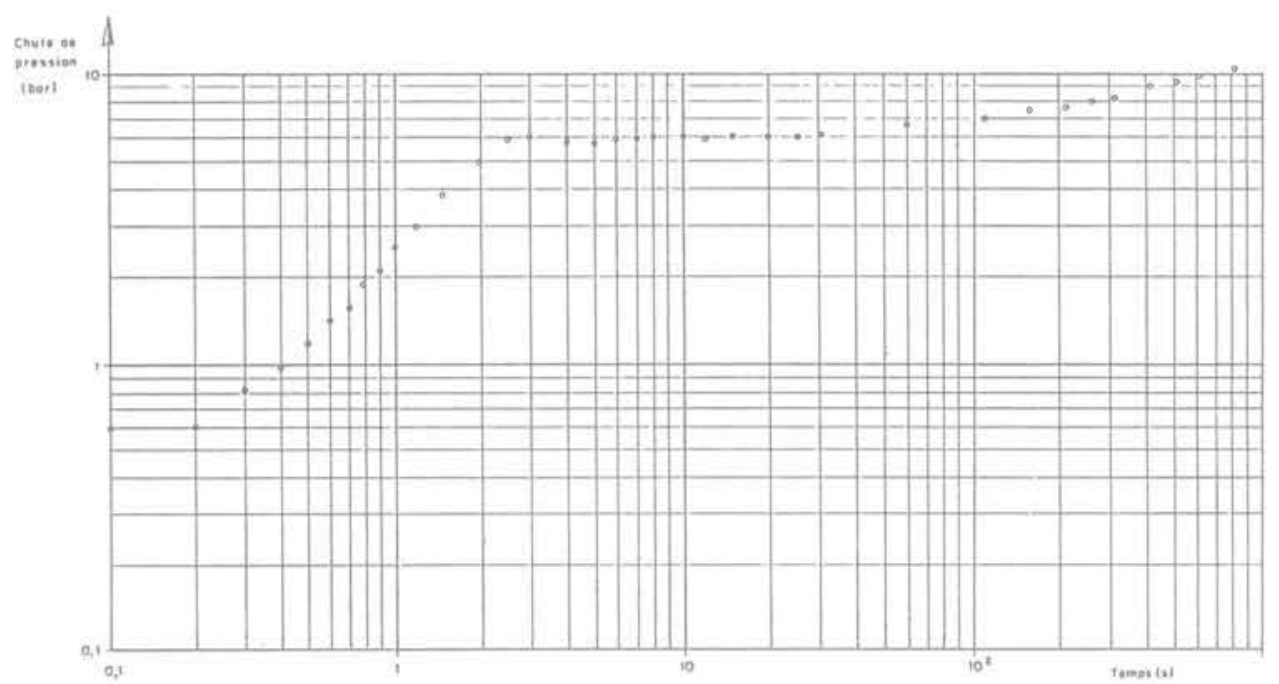

Fig. 9 Exemple de chute de pression après injection dans une fracture verticale

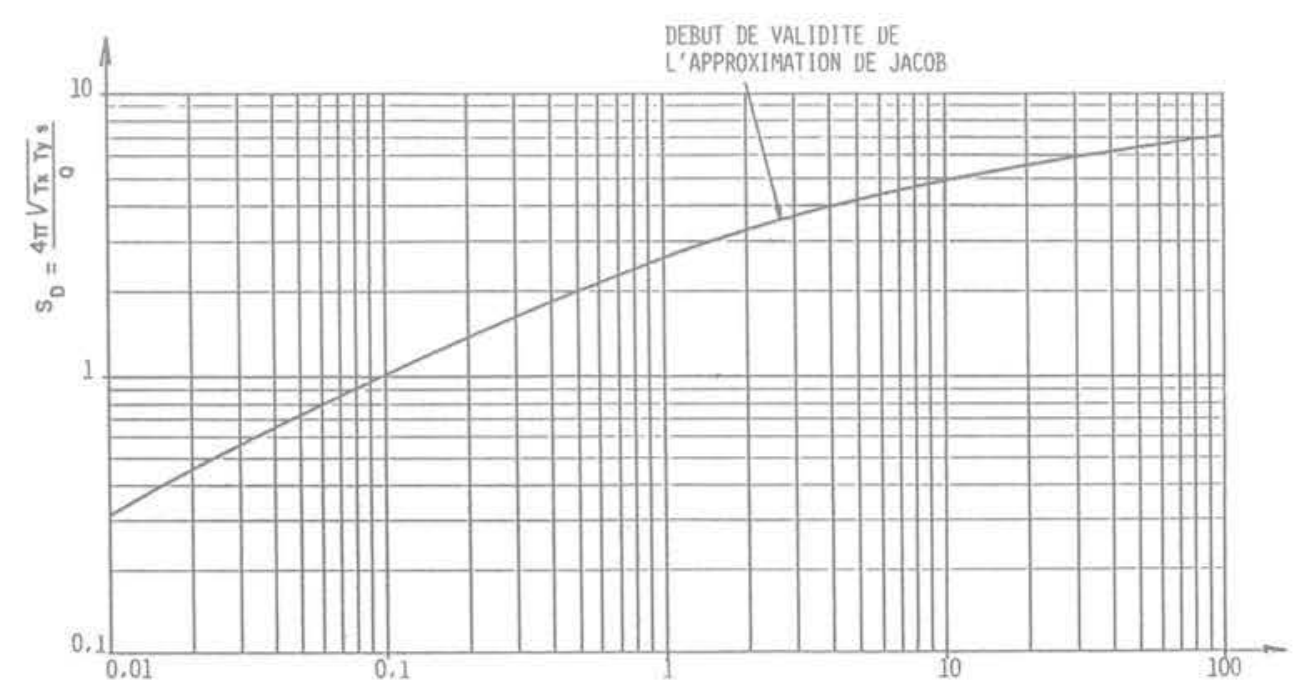

Fig. 10 Courbe type pour une fracture verticale, d'après Gringarten et Ramey. $t_{D}=T x . t / S . x f^{2}$ 
paramètres qui interviennent dans les phénomènes étudiés, qu'il soit possible d'aboutir à la mise au point de méthodes simples, utilisant par exemple des abaques, et permettant des interprétations faciles de ces essais.

En fait, la seule méthode qui apparaisse praticable est une méthode d'identification qui consiste, en utilisant un modèle, et en partant de valeurs estimées à priori pour les paramètres inconnus, à modifier par étapes ces paramètres jusqu'à obtenir une reproduction suffisamment satisfaisante, par le modèle, des phénomènes observés. II est clair qu'une telle méthode peut aboutir dans le cas le plus général a une infinité de solutions. Une bonne compréhension des phénomènes physiques en jeu et une critique sévère des hypothèses faites et des résultats obtenus doit néanmoins permettre d'aboutir à une solution qu'on pourrait qualifier de "la plus vraisemblable".

La question peut être posée de savoir pourquoi, puisqu'on sait encore mal interpréter les résultats d'essais à forte pression, on ne se contente pas d'essais à faible pression pour lesquels les schémas théoriques classiques sont utilisables.

La raison en est que, s'agissant de milieux très peu perméables, I'utilisation de faibles pressions ne permet pas d'obtenir des débits dinjection qui soient mesurables même avec les dispositifs les plus sensibles.

\subsection{Technologie des essais}

II est clair que l'abaissement du seuil de mesure des perméabilités en sondage résulte fondamentalement de progrès technologiques. débitmètres à turbine, montés en parallèle et couvrant à eux trois la gamme de 0.02 à $50 \mathrm{k} / \mathrm{mn}$.

La figure 11 représente le schéma de l'installation utilisée à Kimberley (cf. tableau II).

II s'agit d'une installation très simple, aux performances moyennes, qui ne peut pas être utilisée pour des terrains de perméabilité inférieure à $5 \cdot 10^{-8} \mathrm{~m} / \mathrm{s}$. La pompe d'injection utilisée, de type centrifuge, ne permet pas de dépasser $1 \mathrm{MPa}$ en pression. La mesure des débits à l'aide de compteurs volumétriques a une précision limitée et interdit la détermination des débits instantanés. Enfin, les manomètres à cadran, eux non plus, nautorisent pas en général une précision très élevée.

En outre, toutes les mesures étaient faites manuellement. Un tel systeme, de mise en œuvre facile et peu onéreuse, était toutefois parfaitement adapté à la gamme des perméabilités à déterminer $\left(2 \cdot 10^{-5}\right.$ à $2 \cdot 10^{-7} \mathrm{~m} / \mathrm{s}$ ). Tout autres sont les moyens à mettre en cuvre quand on cherche à déterminer des perméabilités qui peuvent être 1000 fois plus faibles.

La figure 12 représente une installation utilisée pour des mesures dans cette gamme de perméabilités. L'injection est réalisée à l'aide d'une pompe triplex munie d'un changement de vitesses, équipée d'une décharge à pression constante et d'accumulateurs antipulsatoires fonctionnant dans différentes gammes de pression et supprimant les à-coups de pression dus au fonctionnement de la pompe. Les obturateurs, très robustes, peuvent être gonflés par un tube indépendant, à plus de $30 \mathrm{MPa}$.

Les pressions d'injection peuvent atteindre $15 \mathrm{MPa}$. La très faible perméabilité des terrains impose de pouvoir injecter et mesurer avec une bonne précision des débits aussi petits que $0,02 \mathrm{l} / \mathrm{mn}$. Le dispositif de mesure de ces débits est donc constitué par trois

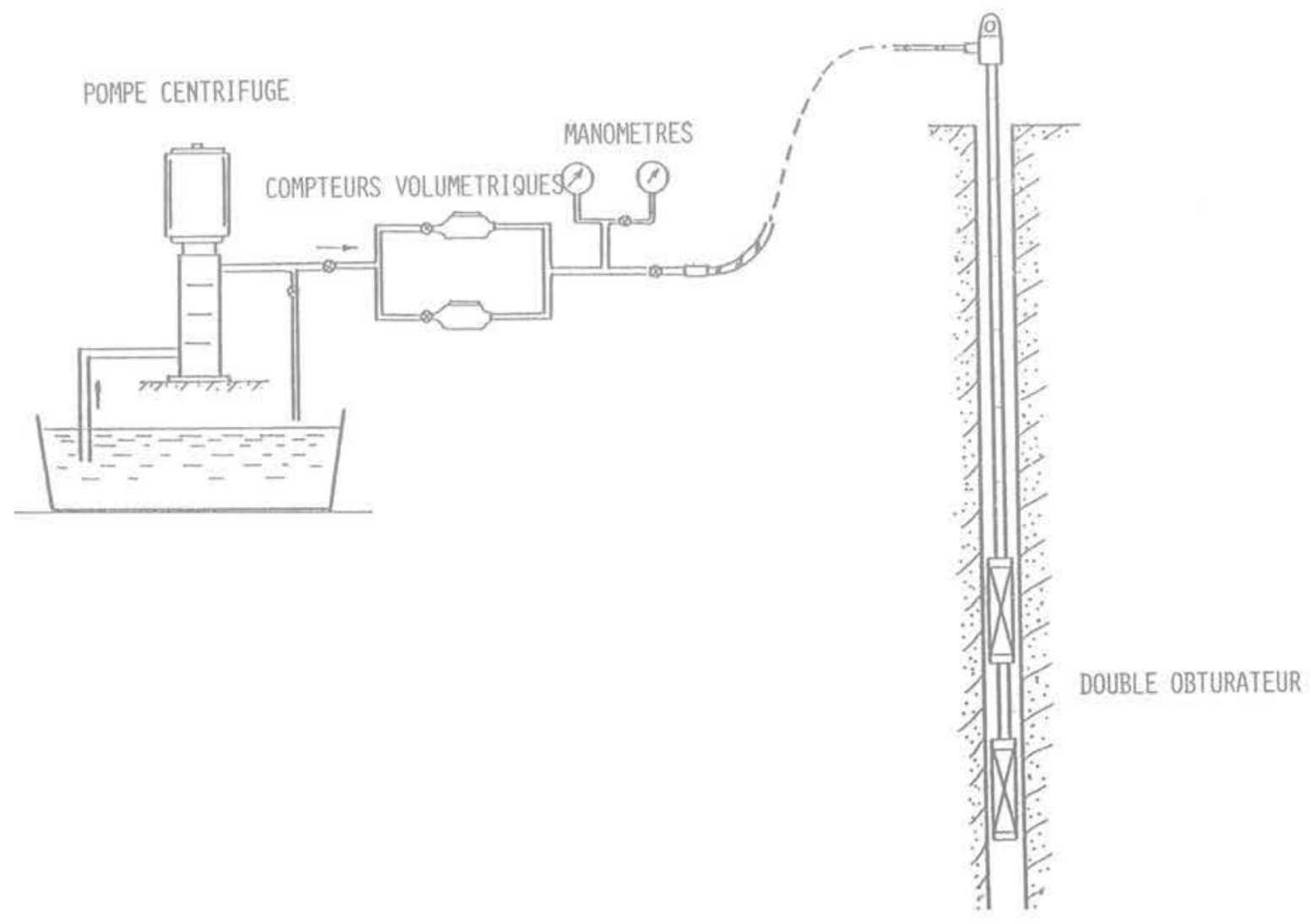

Fig. 11 Dispositif d'injection "classique» 


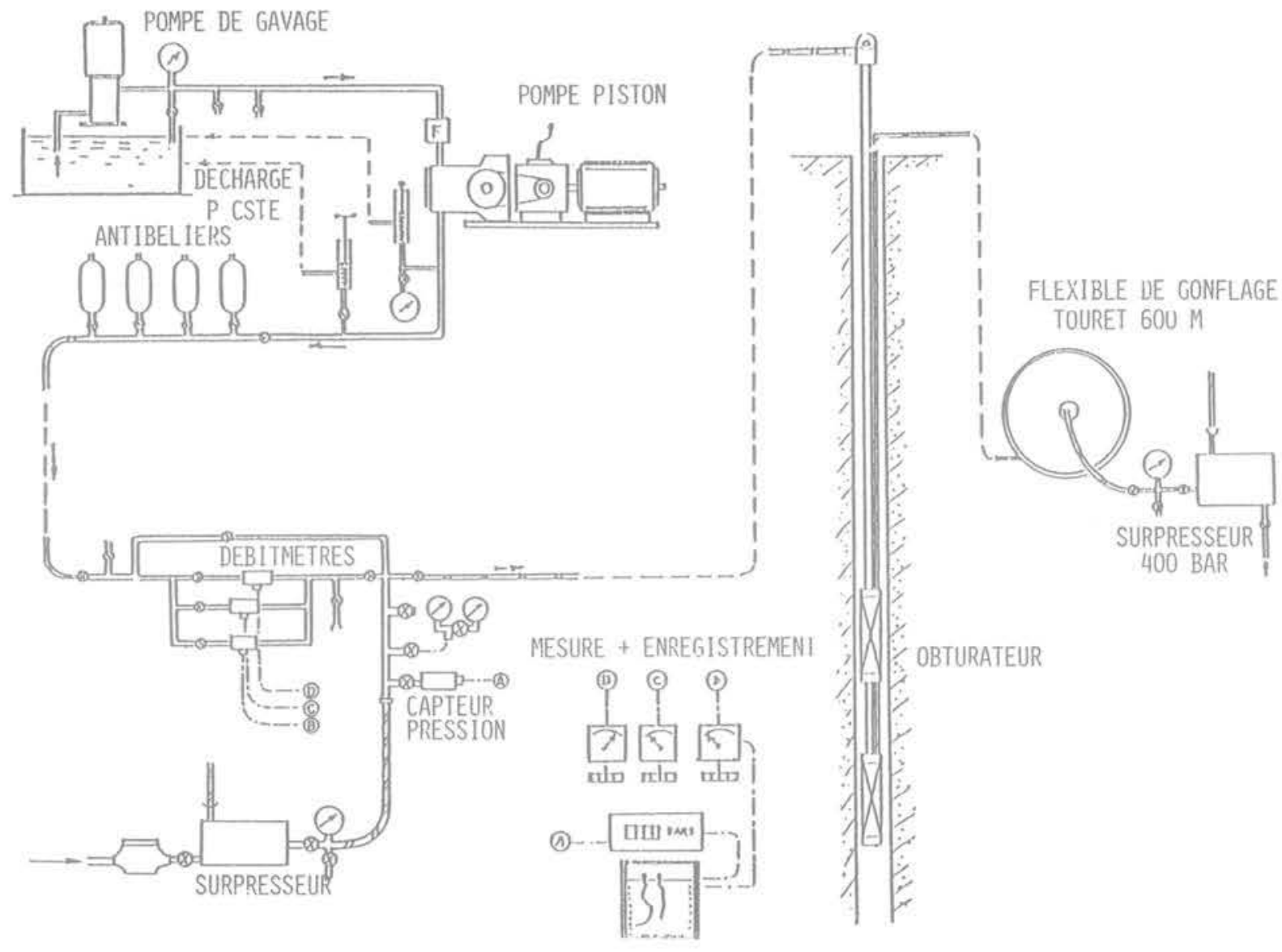

Fig. 12 Dispositif d'injection en milieu très peu perméable

La pression est mesurée en tête par un capteur de pression. Elle est corrigée des pertes de charge, au demeurant très faibles compte tenu de la faible valeur des débits enregistrés et du gros diamètre des tiges d'injection.

Tous ces appareils sont reliés, par l'intermédiaire de leur conditionnement, à un enregistreur analogique multipiste, les valeurs instantanées de la pression et du débit étant par ailleurs affichées sur des voltmètres, à titre de contrôle.

C'est essentiellement ce dispositif extrêmement précis et sensible qui a permis de réaliser un bond en avant dans la mesure des très faibles perméabilités. II va de soi que ces mesures nécessitent également que l'appareillage descendu dans le forage (obturateurs. train de tiges par lequel est envoyée l'eau dans la chambre d'injection, système de gonflage des obturateurs) soit du matériel de haut de gamme et qu'en particulier il ne puisse pas se produire la moindre fuite au contact des obturateurs et de la paroi du forage.

\subsection{Exemple de résultat obtenu}

La figure 13 présente un exemple de profil de perméabilité réalisé sur un sondage incliné au Portugal, à l'aide de la méthode décrite précédemment.

Ce profil a été réalisé en associant les résultats provenant d'un pompage d'essai, intéressant toute la hauteur du sondage, et des injections entre obturateurs ou sous un obturateur simple, la chambre d'injection étant alors délimitée par le fond du trou et la base de l'obturateur.
Ce profil est particulièrement intéressant par le fait qu'il illustre les deux phénomènes mentionnés en 3.1 : on constate en effet une décroissance marquée de la perméabilité avec la profondeur cependant que localement une zone faillée donne lieu à une augmentation brutale de celle-ci.

\section{Conclusion}

La présente communication avait pour but d'exposer la démarche utilisée désormais au B.R.G.M. pour parvenir à la caractérisation des perméabilités des milieux rocheux fracturés.

Cette démarche associe une étude poussée de la fracturation, qui permet de déterminer l'anisotropie de perméabilité, avec des essais à l'eau en sondages dont la sophistication est fonction de la valeur des perméabilités à mesurer. Quoique constituant un net progrès par rapport aux méthodes en vigueur précédemment, cette démarche reste encore imparfaite.

En ce qui concerne la dètermination du degré et de l'orientation de l'anisotropie, la méthode proposée est encore grossière et, bien que l'utilisation de différentes pondérations sur chacun des exemples présentés n'ait pas fait varier considérablement ses résultats, on ne peut se satisfaire vraiment de considérer, même statistiquement, que les différentes familles de fractures recoupant un massif présentent les mêmes propriétés en ce qui concerne la circulation de l'eau. Les méthodes permettant de caractériser simplement, sur affleurement. les propriétés hydraulique d'une fissure restent à inventer. 


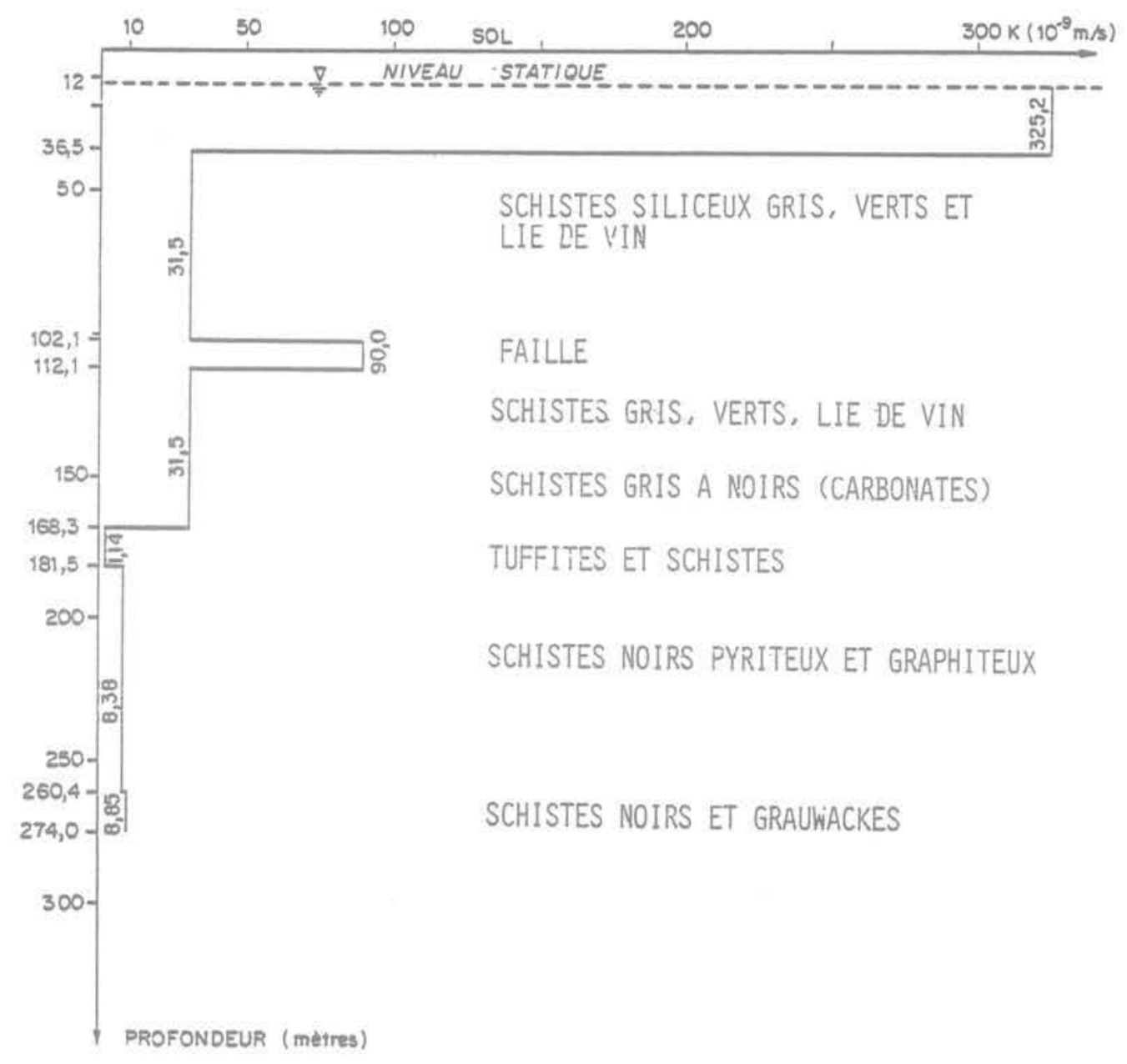

Fig. 13 Exemple de profil de perméabilité

En outre, sur le plan théorique, la méthode devra être améliorée par la prise en compte de l'absence de continuité de la plupart des fissures réelles.

De même, la méthode de calcul proposée nécessiterat-elle, avant de pouvoir être considérée comme sûre, de voir ses résultats confirmés par des déterminations résultant d'essais in situ, sur un nombre suffisant de cas. Un programme de recherches est en cours sur ce sujet.

En ce qui concerne les essais in situ, on a vu que les moyens théoriques permettant de tirer le meilleur profit de leurs résultats étaient encore insuffisants et que des progrès importants pouvaient encore être réalisés dans ce domaine.

II faut noter à ce sujet que les méthodes d'interprétation utilisées actuellement ne prennent pas en compte, ou prennent en compte de façon peu satisfaisante, l'anisotropie du milieu, ce qui fait que le lien entre les deux approches complémentaires décrites dans cette communication se fait encore de façon quelque peu empirique.

II s'agit là aussi d'un domaine sur lequel un important programme de recherches, incluant des essais d'injection avec observation des pressions dans des piézomètres permettant de reconstituer leur distribution à trois dimensions est en cours.

Ce programme devrait permettre de combler la lacune mentionnée plus haut.

\section{Références bibliographiques}

[1] LOUIS C. (1976) Introduction a I'hydraulique des roches. Thèse Doct. ès Sc. Paris VI.

[2] THEIS C.V. (1935) The relation between the lowering of the piezometric surface and the rate and duration of discharge of a well using groundwater storage. T.A.G.U., 1935, 619-24.

[3] JACOB C.E., LOHMAN S.W. (1952) Non steady flow of a well of constant drawdown in an extensive aquifer. T. A. G. U., août 1952, 559-69.

[4] BOULTON N.S. (1954) L'abaissement non permanent de la nappe phréatique près d'un puits de pompage dans une formation indéfinie. Proc. Inst. Civ. Eng. part. III, vol. 3, août 1954, 564-579.

[5] GRINGARTEN A.C., RAMEY H.J. (1974) Unsteady state pressure distribution created by a well with a single infinite conductivity vertical fracture.

Unsteady state pressure distribution created by a well with a single horizontal fracture, partial penetration, or restricted entry. Soc. Pet. Eng. J. août 1974.

[6] FEUGA B., NOYER M.L., THIERY D. (1980) Well testing of low to very low permeability fractured media A review. In "Advances in European geothermal research ". Proc. $2^{\text {nd }}$ Int. Seminar Geothermal Energy research. 1980, Strasbourg. 